\title{
Public investment as commitment
}

\author{
Reyer Gerlagh and Matti Liski*
}

August 172011

\begin{abstract}
Should public assets such as infrastructure, education, and the environment earn the same return as private investments? We consider if time-inconsistent decisionmakers can gain from institutions that enforce cost-benefit rules on large projects that influence the economy as a whole. Long-term public investments provide commitment to current preferences, leading to investment biases in such assets. The institutionalized cost-benefit prudence eliminates the biases but we show that this behavioral rule has no general social value: it implements Pareto efficiency if and only if preferences are time-consistent, and decreases welfare otherwise. We find that the long-term cost-benefit prudence is fundamentally about income transfers to the future, implying that efficient behavioral rules should target savings directly rather than the division of current investment resources.
\end{abstract}

(JEL classification: H43; H41; D61; D91; Q54; E21. Keywords: public investments, cost-benefit analysis, inconsistent preferences)

\section{Introduction}

Cost-benefit analysis (CBA) as a way to bring government projects and programs under public scrutiny is a feature of good governance which most economists agree on. CBA is routinely applied to individual small projects but increasingly also to large programs that influence the economy as a whole as, for example, the Stern Review on

\footnotetext{
${ }^{*}$ Gerlagh <r.gerlagh@uvt.nl $>$ is at the Economics Department of the Tilburg University. Liski $<$ matti.liski@aalto.fi $>$ is at the Aalto University School of Economics, Helsinki. We thank Cedric Argenton, Shelby Gerking, Mads Greaker, Robert Hahn, James K. Hammitt, Gisle James Natvik, Sjak Smulders, Christian Traeger, Marty Weitzman, Aart de Zeeuw, and the participants in seminars at CESifo, ETH Zurich, HECER Helsinki, Statistics Norway, Tilburg, and Toulouse for discussions and comments.
} 
climate change (2006) has illustrated. ${ }^{1}$ Recently, the financial crisis has increased the demand for economy-wide analysis of costs and benefits of public spending and regulations (e.g., Rogoff 2010, and Hanson et al. 2011). Such economy-level CBA exercises require an integrated assessment of how to best allocate overall resources between public and private uses, introducing a complication not present in the small-project cases: large programs influence the direction of the economy over time and thus choices feasible in the future. Today's investments in energy-supply infrastructure shape the conceivable future technology choices, similarly as public transport and city planning mold the future transportation solutions. Public education not only influences the productivity of the population but also alters the overall set of activities that can be considered in the future. Future biodiversity depends on current decisions to establish and maintain large natural parks but this may prevent us from creating wealth in some other form. These public choices allow partial commitment to today's preferred course of actions, while the value generated by the choices depends also on the preferences in the future which may not be congruent with those today. In this paper, we consider if time-inconsistent decision-makers can gain from institutions that enforce "cost-benefit prudence" on large projects that influence the economy as a whole.

Inconsistencies that we have in mind can arise from time-variant preferences (Strotz, 1956), intergenerational altruism (Phelps and Pollak, 1968), or self-control problems (Laibson, 1997). In climate change, it seems compelling to argue that we may not want to distinguish between the welfare of generations 100 and 101, although we discount that of the near-future generations; the future benefits are then converted to present values at discount rates declining with the time horizon, which introduces inconsistencies into climate policies (Karp 2005). ${ }^{2}$ Our point of departure is the observation that timeinconsistent decision-makers value assets not only for the net benefits they generate, but also for the commitment they provide (Laibson, 1997). Intuitively, a decision-maker who values commitment provided by the long-term asset ends up "over-investing" in it, when allowed to freely choose the division of savings between long-term and short-term uses.

One way to view the institutionalized cost-benefit prudence is to think of a budget office scrutinizing the public uses of resources, and enforcing the requirement that public

\footnotetext{
${ }^{1}$ While the Review is the most comprehensive economic report on the climate problem, there is a large literature considering the cost-effectiveness of climate policies; see Nordhaus (1993) for an early contribution.

${ }^{2}$ Non-constant discount rates can also result from aggregation over heterogenous individuals (Gollier and Zeckhauser, 2005, Lengwiler, 2005), or from uncertainty (Weitzman, 2000, Gollier, 2002) in conjunction with consistent preferences. We come back to this question in the concluding section.
} 
investments should earn at least the same comparable return as private investments. ${ }^{3}$ The budget office thus allows decision makers to freely choose allocations over time but, through the return-requirement rule, sets up an institutional constraint on how resources can be divided between public and private uses. While such rules are easy to advocate and therefore may arise as a "social contract" characterizing good public governance, it is not clear that the simple cost-benefit rules have social value in the setting we have described.

Casting the analysis in a Ramsey saving-problem where savings are allocated between the traditional neoclassical capital and long-term public assets, ${ }^{4}$ and where preferences are as in the Phelps-Pollak-Laibson framework, we find that institutionalized cost-benefit rules have no general social value: they implement Pareto efficiency if and only if preferences are time-consistent, and decrease welfare otherwise. Indeed, multi-generation (multi-self) Pareto efficiency - a natural concept for efficiency in this context - cannot follow from one rule for the division of assets between different maturities in a closed economy. ${ }^{5}$ Intuitively, imposing the cost-benefit rule as an institutional constraint removes the investment biases of inconsistent decision-makers, but the rule also removes the commitment value built into the public assets, thereby reducing the overall value of savings as a channel for transferring wealth to the future; the rule limits, e.g., the agents' altruistic plans for the future.

The analysis leads us to conclude that the long-term cost-benefit problem is fundamentally an intertemporal income-transfer problem where efficient rules should target savings directly. While rules for savings are much harder to put on as a simple principle or to delegate to the budget office for implementation, we show that welfare-improving and self-enforcing savings rules have a simple formal structure. In contrast to stand-alone

\footnotetext{
${ }^{3}$ see, e.g., Nordhaus 2007 for a discussion of this return requirement for public investments.

${ }^{4}$ The former capital can be interpreted as resulting from the aggregation of individual decisions and is thus private by nature, while the latter type of capital is public by assumption. We abstract from the aggregation and political economy aspects of the public decisions in order to pinpoint the allocative distortions not solved by CBA even in the representative agent framework. In this closed economy, the capital stock produces endogenously the rate-of-return requirement, or the opportunity cost, of the public investments; such an approach is needed, for example, in the climate context where the policy has an effect on the growth path of the economy (see, e.g., Weitzman 2007 and Nordhaus 2007).

${ }^{5}$ Since we focus on multi-generation welfare comparisons where inconsistencies can arise, e.g., from altruism rather than individuals' behavioral anomalies, we work with the multi-generation Pareto criterion. See Bernheim and Rangel (2009) for an alternative concept and its relationship to the Pareto criterion. We briefly discuss Bernheim and Ragel's definition of a weak welfare optimum in connection to our definition of Pareto efficiency.
} 
cost-benefit rules, savings rules target the source of the problem directly, and thereby reduce the agents' need to use long-term assets for commitment purposes, which explains why such rules also reduce the investment biases of inconsistent decision-makers. In other words, as agents can interact only in the order of their appearance in the time line, they cannot transfer wealth directly to the intended beneficiary represented in the welfare function and the allocation without any policy measures will be inefficient. Enforcing productive efficiency through the cost-benefit rule worsens the problem of the incomplete income transfers, whereas savings rules target the market imperfection directly.

The welfare we consider depends on both current utilities and those of the future generations. ${ }^{6}$ To define sharply the connection between the cost-benefit rule, inconsistent preferences and welfare, we consider whether the equilibrium is observationally consistent with a fictitious (consistent-preferences) "Planner", i.e., an agent who may not represent the actual agent population but who would consider the equilibrium allocation optimal. It is well-known that the hyperbolic discounting models popularized by Laibson (1997), and also O'Donough and Rabin (1999), and Barro (1999) can be interpreted this way: the equilibrium paths of these models maximize a utility stream for some sequence of utility weights but these weights need not represent the underlying (multi-self) preferences.

We find that enforcing the institutionalized cost-benefit rule establishes observational equivalence with a Planner, but the Planner does not represent the population, and Pareto efficiency (or improvement) does not follow from the rule. The question of whether the Planner represents the population provides a direct check for efficiency in this potentially complicated behavioral environment. It also allows us to analyze institutional designs for policy making, e.g., rules on total savings, potentially making the Planner representative for the agent population. Note that this abstraction of the Planner does not entail an imposed dictatorial social welfare function on the economy, but rather it helps to look for policies generating outcomes that are consistent with some welfare objective. ${ }^{7}$ In our view, the approach provides a basis for a positive approach to the optimal institutional design.

We first present a simple three-period example to illustrate the main results. We then

\footnotetext{
${ }^{6}$ In principle, welfare for a given generation can look backwards and forwards in time, i.e., depend also on the utility levels in the past (in a different setting, Caplin and Leahy (2004) consider such a criterion).

${ }^{7}$ In this sense we depart, for example, from Asheim (1988) who imposes an ethical principle (Ralwsian maximin criterion) on each generation to study the intergenerational conflict as a subgame-perfect equilibrium.
} 
extend the framework to an infinite number of periods, ${ }^{8}$ which is needed for the analysis of self-enforcing savings rules and for a more flexible analysis of our results in relation to the persistence of the public asset. For illustrational purposes we connect our analysis to the literature on discounting and optimal climate change policy.

\section{A three-period model}

\section{$2.1 \quad$ The setting}

We first consider three generations, living in periods $t=1,2,3$. In each period, consumers are represented by an aggregate agent having a utility function and production technology. Consumption programs $(\mathbf{c}, q)=\left(c_{1}, c_{2}, c_{3}, q\right) \in A=A_{1} \times A_{2} \times A_{3} \times A_{q}$ (nonempty intervals) constitute a consumption level for each generation and the final asset $q$ to the last generation. Generations are assumed to have the following simple welfare representation

$$
\begin{aligned}
& w_{1}=u_{1}\left(c_{1}\right)+\rho\left[u_{2}\left(c_{2}\right)+\theta\left[u_{3}\left(c_{3}\right)+v(q)\right]\right] \\
& w_{2}=u_{2}\left(c_{2}\right)+\sigma\left[u_{3}\left(c_{3}\right)+v(q)\right] \\
& w_{3}=u_{3}\left(c_{3}\right)+v(q),
\end{aligned}
$$

where all utility functions $u_{t}$ and $v$ are assumed to be continuous and, in addition, strictly concave, differentiable, and satisfying $\lim _{c \rightarrow 0} u_{t}^{\prime}=\infty$ and $\lim _{q \rightarrow 0} v^{\prime}=\infty$. For interpretation, we assume that parameters $\rho, \theta, \sigma \in[0,1]$ are discount factors, although this is not necessary in this three period model. Inconsistent preferences are identified by $\theta \neq \sigma$, i.e., the first and second generations disagree on the relative weight given to the last generation's utility. When $\theta>\sigma=\rho$, the near future is discounted more than the far future. Following Phelps and Pollak (1968) or, e.g., Saez-Marti and Weibull (2005) this can be interpreted as pure altruism towards the last generation, or alternatively as lack of (governmental) self control (Laibson, 1997). ${ }^{9}$ For completeness, we also allow for

\footnotetext{
${ }^{8}$ However, the equilibrium analysis requires restrictions not present in three periods (see also Krusell et al. 2002, and Karp 2005, and 2007).

${ }^{9}$ We can obtain the common $\beta, \delta$ model as in Phelps-Pollak-Laibson if $\rho=\sigma$, by defining $\beta=\rho / \theta$ and $\delta=\theta$. Then, $w_{1}=u_{1}+\beta \delta u_{2}+\beta \delta^{2} u_{3}$ and $w_{2}=u_{2}+\beta \delta u_{3}$. Inconsistencies are identified by $\beta<1$, corresponding to $\theta>\sigma$ in our case. For our purposes, it is slightly more straightforward to name the long-run weights as $\theta$ and $\sigma$. The weight $\rho$ allows some freedom in terms of interpretations (e.g. the length of a period may be different for $t=1,2,3)$ but is inconsequential for the consistency of the preferences.
} 
the case $\theta<\sigma$. This could represent a situation where the representative agent looks one period ahead with less interest in the future further away.

Generations consider choices in a convex consumption possibility set $A \subseteq R_{+}^{4}$. The consumption possibilities are determined by a strictly concave neoclassical production function $f_{t}\left(k_{t}\right)$, where $k_{t}$ is the capital stock they receive from the previous generation. The first generation starts with a capital stock $k_{1}$, and produces output which can be used to consume $c_{1}$, to invest in capital for the immediate next period $k_{2}$, or to invest in a durable asset for the third period, $q$ :

$$
c_{1}+k_{2}+q=f_{1}\left(k_{1}\right)
$$

The second agent starts with the capital stock $k_{2}$, which produces output $f_{2}\left(k_{2}\right)$, and can use its income to consume $c_{2}$, or to invest in capital for the third period $k_{3}$ :

$$
c_{2}+k_{3}=f_{2}\left(k_{2}\right)
$$

In this simple example, we abstract from possibilities of the second consumer to invest in the durable asset $q$. In the next section, we consider a more flexible form to describe dynamics for the public good. The third consumer derives utility from its consumption,

$$
c_{3}=f_{3}\left(k_{3}\right)
$$

and from the inherited durable asset $v(q)$.

\subsection{Welfare and efficiency}

Consider an allocation $(\mathbf{c}, q)$ that is Pareto efficient for welfare levels $\left(w_{1}^{*}, w_{2}^{*}, w_{3}^{*}\right)$ defined in (1)-(3). If we maximize $w_{1}$, subject to the constraints $w_{2} \geq w_{2}^{*}$, and $w_{3} \geq w_{3}^{*}$ and feasibility constraints (4)-(6), then we must find the same allocation, and non-negative Lagrange multipliers $(\alpha, \beta) \in R_{+}^{2}$ for the welfare constraints. That is, the Pareto efficient allocation is also the solution of a welfare program maximizing

$$
\begin{aligned}
W(\mathbf{c}, q) & =w_{1}+\alpha w_{2}+\beta w_{3} \\
& =u_{1}\left(c_{1}\right)+(\rho+\alpha) u_{2}\left(c_{2}\right)+(\rho \theta+\alpha \sigma+\beta)\left[u_{3}\left(c_{3}\right)+v(q)\right]
\end{aligned}
$$

subject to (4)-(6). ${ }^{10}$ The conclusion also holds the other way around: any solution to a welfare maximization program with some $(\alpha, \beta) \in R_{+}^{2}$ is Pareto efficient. Strict

\footnotetext{
${ }^{10}$ We notice that a Pareto optimum as defined by Bernheim and Rangel (2009, Corollary 2) need not maximize the above welfare program. Bernheim and Rangel's condition defines a Pareto optimum
} 
concavity of the production and utility functions ensures the uniqueness of the allocation. Therefore, we can associate any Pareto efficient allocation with a pair of non-negative welfare weights $(\alpha, \beta) \in R_{+}^{2}$, and also with a "Planner" whose objective function is the corresponding $W(\mathbf{c}, q)$ giving some non-negative weight for all generations. ${ }^{11}$ If and only if weights are non-negative, we say that the Planner is representative, and the allocation is Pareto efficient. ${ }^{12}$ We emphasize that the abstraction of the Planner is merely a tool for welfare analysis, and not intended to introduce preferences over allocations - an allocation is judged "good" when a representative Planner can be attached to it, or a policy is an improvement if the implied allocation comes closer to being representative. Thus, parameters $\alpha, \beta$ do not represent altruism of an outside agent or institution because they arise from the equilibrium outcome, not the other way around. Consumers are the only source of altruism, and we take an agnostic approach to its size and nature as captured through $\rho, \theta$ and $\sigma{ }^{13}$

Instead of the above welfare aggregator, we can characterize the Planner defined through a utility aggregator. For this, consider some feasible equilibrium allocation (c, $q$ ) implying a stream of utilities $\left(u_{1}^{*}, u_{2}^{*}, u_{3}^{*}, v^{*}\right)$, such that the allocation maximizes

$$
U(\mathbf{c}, q)=u_{1}\left(c_{1}\right)+\alpha^{\prime} u_{2}\left(c_{2}\right)+\beta^{\prime}\left[u_{3}\left(c_{3}\right)+v(q)\right] .
$$

through a lower bound for $w_{1}$. An important distinction with multi-self efficiency is that the BernheimRangel ordering cannot distinguish between two utility sequences that are close, even when one is strictly above the other.

Consider two sequences $A$ and $B$, both with constant utility, sequence $A$ slightly above sequence $B$. Our multiple-self efficiency unambiguously ranks $A$ above $B$. Bernheim and Rangel (2009) show that $A$ and $B$ cannot be ordered based on choices. They construct an auxiliary sequence $C$ (e.g. high utility for the second agent, low utility for the first and third agent) such that the first agent prefers $A$ over $B$ over $C$, while the second agent prefers $C$ over $A$ over $B$. If the first agent has to choose between $\{A, C\}$ and $\{B\}$, foresight of the second agent's choice implies he will choose $\{B\}$. Therefore, from a choice perspective, $B$ is not inferior to $A$.

${ }^{11}$ Note that here the welfare is determined in a forward-looking manner but we could also define backward-looking welfare weights as in Caplin and Leahy (2004).

${ }^{12}$ We rule out allocations where the weight on $w_{1}$ equals zero. The weight on $w_{1}$ approaches zero, in relative terms, when at least one of the other weights becomes sufficiently large.

${ }^{13}$ Using the wording of the literature, one may call positive welfare weights $\alpha, \beta$ as "pure altruism" of the Planner. However, altruism of the Planner is not equivalent to that of the agents, so that while individual agents may give pure positive altruistic weights on future welfare levels, the equilibrium outcome need not imply positive welfare weights. Saez-Marti and Weibull (2005) describe the agents' discount functions consistent with pure altruism; in our case, the condition is $\theta>\sigma$. As we will see, in equilibrium this implies purely altruistic agents but negative welfare weighting, thus the Planner is not "altruistic". 
for some positive utility weights $\left(\alpha^{\prime}, \beta^{\prime}\right) \in R_{+}^{2}$. If such positive weights exist, the allocation is observationally equivalent to a Planner's optimum with objective $U(\mathbf{c}, q)$, or shortly, the equilibrium is Planner-equivalent - if and only if the utility weights are positive, we say that the allocation is Planner-equivalent. However, the Planner defined this way need not be representative; as in "hyperbolic" discounting cases, the observational Planner implied by the equilibrium may represent none of the agents (Barro, 1999), or more normatively, the utility weights could represent long-run preferences (O'Donough and Rabin, 1999). In our multi-generation context, we find it natural to look for a Planner that is representative, but it will be of interest to discuss also the weaker concept of Planner-equivalence because only the latter concept will be implied by the cost-benefit requirement. Whereas Pareto efficiency immediately implies Planner-equivalence, the converse is not immediate:

Remark 1 A Planner-equivalent allocation, utility weights $\alpha^{\prime}$ and $\beta^{\prime}$, is representative (Pareto-efficient) if and only if

$$
\begin{aligned}
& \alpha^{\prime} \geq \rho \\
& \beta^{\prime} \geq \rho \theta+\left(\alpha^{\prime}-\rho\right) \sigma .
\end{aligned}
$$

It is clear that if the stated inequalities hold, there are positive welfare weights $(\alpha, \beta)$ corresponding to the utility weights $\left(\alpha^{\prime}, \beta^{\prime}\right)$, and respecting the original preference structure (1)-(3). The "only if" part follows from the observation that if one of the inequalities is not met, then one of the implied welfare weights $\alpha$ or $\beta$ must be negative. Intuitively, the Planner represents all generations only if it gives weights on future utilities that are sufficiently large so that future generations receive non-negative welfare weights in addition to those coming directly from previous generations, e.g., due to altruism.

This notion of a Planner will be useful because it provides direct access to the welfare properties of the equilibria considered below. First, in the equilibrium without the costbenefit requirement, we can show that there is no Planner at all, i.e., no implied utility weights $\left(\alpha^{\prime}, \beta^{\prime}\right)$ exist, and consequently Pareto efficiency can immediately be ruled out. Second, the cost-benefit rule implies Planner-equivalence, and vice versa, but the Planner is not representative, i.e., the implied welfare weights are not positive. Finally, in the end of the paper, (for the infinite horizon model) we discuss savings rules that imply a representative Planner, i.e., these savings rules imply Pareto efficiency. 


\subsection{Efficiency and the cost-benefit rule}

We describe now how the cost-benefit rule follows from Pareto efficiency. For any such allocation, we have $\alpha^{\prime}=\rho+\alpha>0$ and $\beta^{\prime}=\rho \theta+\alpha \sigma+\beta>0$; for convenience of notation, we use the utility maximization program (9) in this section. The first-order conditions for $\left\{k_{2}, k_{3}, c_{1}, c_{2}, c_{3}\right\}$ tell us that any Pareto efficient allocation satisfies:

$$
1=\left[\frac{\alpha^{\prime} u_{2}^{\prime}}{u_{1}^{\prime}}\right] f_{2}^{\prime}=\left[\frac{\beta^{\prime} u_{3}^{\prime}}{u_{1}^{\prime}}\right] f_{2}^{\prime} f_{3}^{\prime} .
$$

Denote by $M R S_{i, j}>0$ the marginal-rate of substitution of consumptions between periods $(i, j)$ (defined to be positive). Let $R_{i, j}$ denote the (compound) rate of return on capital from period $i$ to $j$. We can then re-express the first-order conditions as the usual consumption-based asset pricing equation:

$$
1=\frac{R_{1,2}}{M R S_{1,2}}=\frac{R_{1,3}}{M R S_{1,3}}
$$

Thus, the marginal rate of substitution equals the return on savings. For the investment in the public asset $q$ to the last generation, the first-order condition requires $u_{1}^{\prime}=\beta^{\prime} v^{\prime}$, which we rewrite as

$$
1=M R S_{1, q}
$$

where $M R S_{1, q}$ is defined between period 1 consumption and $q$. To account for the opportunity cost of transferring period 1 output to the asset $q$, combine $M R S_{q, 3}=$ $M R S_{q, 1} \cdot M R S_{1,3}$ and $M R S_{1,3}=R_{1,3}$ yielding:

$$
1=\frac{R_{1,3}}{M R S_{q, 3}}
$$

This is the consumption-based cost-benefit rule. The benefit of one unit of investment in the long-term asset $q$ is measured in terms of the third-period consumption good. This return to direct long-term investments should equal the opportunity cost determined by the compound return on capital $k$. Under efficiency, the long-term asset $q$ should yield the same return as the capital asset $k$. Noticeably, the cost-benefit rule is neutral with respect to, that is, independent of, weights given to each generation's utility. The cost-benefit rule is a necessary and sufficient test for the existence of a Planner.

Lemma 1 An allocation with strictly positive consumption, capital and public investment is Planner-equivalent, that is maximizes $U(\mathbf{c}, q)$ with positive $\alpha^{\prime}$ and $\beta^{\prime}$, if and only if the cost-benefit rule is satisfied. 
Proof. Necessity of the cost-benefit rule has been established above. For sufficiency, we notice that given the allocation, we can construct positive weights $\alpha^{\prime}$ and $\beta^{\prime}$ from (12) such that with these weights the Planner prefers not to deviate from $\left\{k_{2}, k_{3}, c_{1}, c_{2}, c_{3}\right\}$. The cost-benefit rule then ensures that the first-order condition for $q$ is also satisfied.

The equivalence will be instrumental in our equilibrium analysis. First, if the costbenefit rule is not satisfied, the equilibrium allocation implies that no Planner can exist. We find in the next section that in equilibrium the cost-benefit rule will not hold, so the conclusion for efficiency is immediate. Then, in the following section, we impose the cost-benefit rule as an institutional constraint on the equilibrium. We show that such an equilibrium implies positive utility weights and thus restores the Planner-equivalence, but the implied welfare weights are not all positive unless preferences are consistent.

\subsection{Equilibrium}

Consider now the subgame-perfect equilibrium (SPE) of the game where generations choose consumptions and investments in the order of their appearance in the time line, given the preference structure (1)-(3).

The third agent consumes all capital received and enjoys the long-term asset. The second agent decides on the capital $k_{3}$ transferred to the third agent, given the longterm asset $q$ chosen by the first agent and the capital inherited $k_{2}$. We thus have a policy function $k_{3}=g\left(k_{2}, q\right)$, but for the separable utility specification, second-period investment only depends on the stock of capital received, $k_{3}=g\left(k_{2}\right)$. The policy function $g$ ensures that the following first-order condition is maintained

$$
1=\frac{\sigma u_{3}^{\prime}}{u_{2}^{\prime}} f_{3}^{\prime}
$$

The strict concavity of utility implies consumption smoothing, and thus if the second agent inherits marginally more capital $k_{2}$, the resulting increase in output is not saved fully but rather split between the second and third generation:

Lemma 2 Policy function $g$ satisfies $0<g^{\prime}<R_{1,2}$.

Proof. Substitute the policy function $k_{3}=g\left(k_{2}\right)$ in (16),

$$
\sigma u_{3}^{\prime}\left(f_{3}\left(g\left(k_{2}\right)\right)\right) f_{3}^{\prime}\left(g\left(k_{2}\right)\right)=u_{2}^{\prime}\left(f_{2}\left(k_{2}\right)-g\left(k_{2}\right)\right)
$$

and take the full derivatives with respect to $k_{2}$ to obtain

$$
\sigma g^{\prime}\left(u_{3}^{\prime \prime} f_{3}^{\prime} f_{3}^{\prime}+u_{3}^{\prime} f_{3}^{\prime \prime}\right)=u_{2}^{\prime \prime}\left(f_{2}^{\prime}-g^{\prime}\right)
$$


which leads to

$$
g^{\prime}=\frac{f_{2}^{\prime} u_{2}^{\prime \prime}}{\sigma u_{3}^{\prime \prime} f_{3}^{\prime} f_{3}^{\prime}+\sigma u_{3}^{\prime} f_{3}^{\prime \prime}+u_{2}^{\prime \prime}}<f_{2}^{\prime}=R_{1,2}
$$

as $u_{t}^{\prime \prime}, f_{t}^{\prime \prime}<0$ and $f_{t}^{\prime}, u_{t}^{\prime}>0$.

The first agent decides on consumption and investment in the long-term asset, given the policy function $g$, to maximize its welfare

$$
w_{1}=u_{1}+\rho\left[u_{2}\left(f_{2}\left(k_{2}\right)-g\left(k_{2}\right)\right)+\theta u_{3}\left(f_{3}\left(g\left(k_{2}\right)\right)+\theta v_{3}(q)\right] .\right.
$$

The first-order conditions for investments $k_{2}$ and $q$, respectively, are:

$$
\begin{aligned}
& u_{1}^{\prime}=\rho\left(f_{2}^{\prime}-g^{\prime}\right) u_{2}^{\prime}+\rho \theta f_{3}^{\prime} g^{\prime} u_{3}^{\prime} \\
& u_{1}^{\prime}=\rho \theta v^{\prime} .
\end{aligned}
$$

The equations reflect the fact that the marginal cost of investment, i.e., the marginal utility loss, is the same for both types of investments. Rewriting after substitution of (16) gives $^{14}$

$$
M R S_{q, 3}=\left[\frac{\sigma}{\theta}\left(f_{2}^{\prime}-g^{\prime}\right)+g^{\prime}\right] f_{3}^{\prime} .
$$

This condition is the equilibrium version of the cost-benefit rule (15). To assess the deviation from the rule (15), consider the difference between the equilibrium market return on capital and the public asset. In view of (20), the gap $R_{1,3}-M R S_{q, 3}$ can be written as

$$
f_{2}^{\prime} f_{3}^{\prime}-\left[\frac{\sigma}{\theta}\left(f_{2}^{\prime}-g^{\prime}\right)+g^{\prime}\right] f_{3}^{\prime}=\left(1-\frac{\sigma}{\theta}\right)\left(f_{2}^{\prime}-g^{\prime}\right) f_{3}^{\prime} .
$$

This together with Lemma 2 implies

$$
R_{1,3}-M R S_{q, 3}>0 \text { if and only if } \frac{\sigma}{\theta}<1 .
$$

Thus, in equilibrium, the first agent invests in the long-term asset $q$ up to a point where the rate of return falls short of the rate of return on capital over the same period, if and only if $\sigma<\theta$, i.e., the first agent gives a higher weight to the long-term utility than the second agent. The result has a very simple intuition. The first consumer would like to transfer more wealth to the third consumer, compared with the preferred wealth transfer of the second consumer. This is possible through the asset $q$, and thus the long-term asset is more valuable to the first agent, which is reflected in the lower return requirement. The opposite distortion - too little investment-occurs if $\sigma>\theta$.

\footnotetext{
${ }^{14}$ Note that the marginal rate of substitution between $q$ and $c_{3}$ is independent of weights on utilities, and therefore there is no need to indicate who's preferences are in question.
} 
Proposition 1 If preferences are inconsistent $(\sigma \neq \theta)$, the public investment in the longterm asset does not satisfy the cost benefit rule, i.e., $M R S_{q, 3} \neq R_{1,3}$. The equilibrium return falls short of $R_{1,3}$ iff $\sigma<\theta$.

Proof. Above.

We can immediately conclude:

Corollary 1 The equilibrium is not Planner-equivalent if $\sigma \neq \theta$.

This conclusion follows from Lemma 1 which shows that the allocation can be interpreted as some Planner's allocation (including the representative Planner) if and only if the cost-benefit rule holds. Since the equilibrium deviates from the rule, we cannot find positive welfare weights that would support the equilibrium outcome as Pareto efficient. Let us now consider if the Planner-equivalence can be restored by an exogenous (institutionalized) cost-benefit requirement.

\subsection{Cost-benefit law equilibrium}

A simple suggestion for alleviating the efficiency loss due to the deviation from the costbenefit rule is an intertemporal cost-benefit law requiring that all public investments should earn the same return as private investments. We impose such a restriction as an institutional constraint on the equilibrium behavior - it can be thought of as a budget office scrutinizing the investment plan at the end of each period. The budget office has no preferences, and it simply enforces the cost-benefit requirement, without restricting the choices of each generation in any other way.

In three periods, the law will constrain only the first generation's choices for consumption and investments in the two purposes. Given the policy function $g$ of the second generation, the first generation maximizes

$$
w_{1}=u_{1}+\rho\left[u_{2}\left(f_{2}\left(k_{2}\right)-g\left(k_{2}\right)\right)+\theta u_{3}\left(f_{3}\left(g\left(k_{2}\right)\right)+\theta v_{3}\left(q_{3}\right)\right]\right.
$$

subject to the budget equation and the cost-benefit requirement, i.e., eq. (15) restated,

$$
M R S_{q, 3}=R_{1,3}
$$

While the consumption-based cost-benefit rule (CBR) implies a complicated-looking constraint on the current actions, there is a simple way to model it. Note that the CBR 
reduces the first generation's control of the equilibrium allocation: it can only decide on the total savings as the cost-benefit rule determines the division savings between the two assets. Let $I$ denote the total savings by generation 1 . Now, when facing savings $I$ the budget office needs the imputed equilibrium returns on the two assets in order to allocate the savings among the two assets such that the CBR is satisfied. The imputed returns depend on generation 2's policy function, so the budget office needs to solve the generation 2 problem to fulfill its task of allocating savings for the two purposes. But as the second generation has no time-inconsistency problem, it therefore cannot gain by deviating from the cost-benefit rule. The budget office's task and the second generation's preferences thus run parallel, and we can interpret the equilibrium as one where the budget office at the end of period 1 and the second generation are joined.

Given that the budget office is known to behave this way, we may then solve for the equilibrium behavior under the following budget sets:

$$
\begin{aligned}
c_{1}+I & =f_{1}\left(k_{1}\right) \\
c_{2}+k_{3} & =f_{2}(I-q) \\
c_{3} & =f_{3}\left(k_{3}\right),
\end{aligned}
$$

where $I$ indicates the overall saving of generation $1, q$ is the public investment that the second generation sets apart for the third generation, and $k_{3}$ is the capital stock transferred to generation 3 . Note that this change in the timing of the decision on public investment $q$ leaves the production possibility set of the economy unaltered.

The second generation finds the optimal investments portfolio in the two stocks $k_{2}$ and $q$ under budget constraints (23)-(24) and given wealth from the previous generation $I$ by solving

$$
\max _{k_{3}, q} u_{2}\left(c_{2}\right)+\sigma\left[u_{3}\left(c_{3}\right)+v(q)\right]
$$

leading to equilibrium conditions

$$
\begin{aligned}
u_{2}^{\prime} & =\sigma u_{3}^{\prime} f_{3}^{\prime} \\
u_{2}^{\prime} f_{2}^{\prime} & =\sigma v^{\prime},
\end{aligned}
$$

and thus

$$
\frac{v^{\prime}}{u_{3}^{\prime}}=M R S_{q, 3}=R_{1,3}=f_{2}^{\prime} f_{3}^{\prime} .
$$

We see therefore immediately that the cost-benefit rule will be satisfied, irrespective of the wealth transfer $I$ from generation 1. This is no surprise because, as pointed out above, generation 2 has no time-inconsistency problem. 
While the CBR restores the "productive efficiency" in the public investment, the first generation can still decide on transfer $I$ following its own preferences. It is therefore not clear whether the CBR restores efficiency in terms of welfare. To explore this, consider conditions (26)-(27) defining generation 2's policy functions $g(I)$ and $h(I)$ for capital $k_{3}$ and public investment $q$, respectively. ${ }^{15}$ Using the policies, we can write the continuation value for generation 1 as

$$
V_{2}(I)=u_{2}\left(f_{2}(I-h(I))-g(I)\right)+\theta u_{3}\left(f_{3}(g(I))\right)+\theta v(h(I))
$$

to obtain the return for investment $I$ as

$$
\begin{aligned}
V_{2}^{\prime}(I) & =\left[\left(1-h^{\prime}\right)-g^{\prime}\right] f_{2}^{\prime} u_{2}^{\prime}+\theta f_{3}^{\prime} g^{\prime} u_{3}^{\prime}+\theta h^{\prime} v^{\prime} \\
& =\left[1+\left(\frac{\theta}{\sigma}-1\right)\left(h^{\prime}+g^{\prime}\right)\right] f_{2}^{\prime} u_{2}^{\prime},
\end{aligned}
$$

where the latter line follows from using (26)-(27). Note that $h^{\prime}>0$ and $g^{\prime}>0$. The first generation balances costs and benefits of the transfer by choosing $I$ to satisfy

$$
u_{1}^{\prime}\left(f_{1}\left(k_{1}\right)-I\right)=\rho V_{2}^{\prime}(I)
$$

implying

$$
\alpha^{\prime}=\frac{u_{1}^{\prime}}{u_{2}^{\prime} f_{2}^{\prime}}=\rho\left[1+\left(\frac{\theta}{\sigma}-1\right)\left(h^{\prime}+g^{\prime}\right)\right] \geq 0 .
$$

The equilibrium thus puts this implicit value for the utility weight $\alpha^{\prime}$ in the Planner's program that maximizes the value $U(\mathbf{c}, q)=u_{1}\left(c_{1}\right)+\alpha^{\prime} u_{2}\left(c_{2}\right)+\beta^{\prime}\left[u_{3}\left(c_{3}\right)+v(q)\right]$. Similarly, we have

$$
\frac{\beta^{\prime}}{\alpha^{\prime}}=\frac{u_{2}^{\prime}}{u_{3}^{\prime} f_{3}^{\prime}}=\sigma
$$

so that the implied $\beta^{\prime}$ is

$$
\beta^{\prime}=\sigma \rho\left[1+\left(\frac{\theta}{\sigma}-1\right)\left(h^{\prime}+g^{\prime}\right)\right] \geq 0
$$

We can now state the welfare consequences of the cost-benefit requirement.

Proposition 2 The welfare implications of the institutionalized $C B R$ :

1. The equilibrium with the cost-benefit rule is Planner-equivalent for $\theta \neq \sigma$ and $\theta=\sigma$.

2. The Planner is representative (implements Pareto efficiency) if and only if $\theta=\sigma$.

\footnotetext{
${ }^{15}$ By the assumptions made on the primitives of the model, the policy function are continuous, increasing, and differentiable.
} 
Proof. We have seen in Lemma 1 that the CBR and the concept of a Planner are equivalent. Above we constructed the allocation satisfying the cost-benefit rule, and derived the implied non-negative weights $\left(\alpha^{\prime}, \beta^{\prime}\right)$, without any restrictions on the discount factors. This proves the first item. For the second item, we show that inequalities in Remark 1 can hold if and only if $\theta=\sigma$. Thus, only for consistent preferences are the implied welfare weights non-negative. For inequality (10), note that

$$
\alpha^{\prime}=\rho\left[1+\left(\frac{\theta}{\sigma}-1\right)\left(h^{\prime}+g^{\prime}\right)\right] \geq \rho \Leftrightarrow \theta \geq \sigma .
$$

For inequality (11), substitute (29) and write

$$
\beta^{\prime}=\sigma \alpha^{\prime} \geq \rho \theta+\left(\alpha^{\prime}-\rho\right) \sigma
$$

which simplifies to

$$
\sigma \geq \theta
$$

We see that (10) and (11) are in contradiction unless $\theta=\sigma$, a case in which equalities hold in (31) and (32). If $\theta>\sigma$, then (31) and thus (10) is satisfied but (32) violated. If $\theta<\sigma$, then by (31) condition (10) is violated.

It is worth emphasizing why the CBR equilibrium violates Pareto efficiency. When $\theta>\sigma$, the $\mathrm{CBR}$ equilibrium implies that the welfare weight on the last generation is negative, $\beta<0$. This is intuitive as the first generation would like to transfer more wealth to the last generation but cannot do so due to the CBR. The fact that the first generation is prevented from implementing its altruistic plan for the future distorts the overall savings below the minimum level that supports Pareto efficiency. On the other hand, if $\theta<\sigma$, the implied weight on the middle generation is negative, $\alpha<0$.

Corollary 2 The CBR does not imply a welfare Pareto improvement vis-a-vis the equilibrium without the cost-benefit law.

The reason for this result is simple: the cost-benefit law is only a constraint on the first generation, as it could have implemented such a law without consulting the later generations. Therefore, enforcing the CBR must decrease welfare of the first generation if $\theta \neq \sigma$. If preferences are time-consistent, imposing the CBR has no effect on the equilibrium. In three periods, generation 1 cannot benefit from the later generations' adherence to the CBR, and this is one reason to explore the infinite-horizon model in Section 3. The more general model also facilitates the analysis of self-enforcing savings rules, and more flexible investments in $q$ rather than direct transfers welfare from the first to the last generation. 


\subsection{Discussion}

The main lessons will carry over to the more general model, so we may discuss some policy implications after this preliminary analysis. It should first be emphasized what is not implied by the analysis: we do not want to implicate that fully efficient policies should not satisfy the cost-benefit rule. The cost-benefit requirement is a simple policy rule to advocate and something that could potentially arise as an "intergenerational social contract", characterizing good public governance. We have demonstrated only that the cost-benefit rule, as a stand-alone rule dictating the allocation of resources among alternative uses, cannot internalize all inefficiencies when the overall amount of resources left for the future is open to choice. The core of the welfare inefficiency is that the first generation cannot directly transfer income to any but the immediately next future generation, which is a source of market incompleteness when the agents' objectives are incongruent. The institutionalized cost-benefit rule prevents the use of public assets for altruistic purposes, which then reduces the value of overall savings, thereby adding to the existing intergenerational welfare-transfer distortion. This key problem of the cost-benefit rule has already been discussed by Lind (1995), but qualitatively. In order to benefit all parties the cost-benefit requirement should be accompanied by policy rules steering the savings rate. While we can think of various "golden rules" for the public sector finances (see, e.g., Bassetto and Sargent, 2006), the savings decisions are inherently private, and it is thus less clear if anything as easy to interpret as a rule as the cost-benefit check can be devised for savings. However, in Section 3 we discuss such welfare-improving saving rules.

While we do not provide an explicit political-economy justification for the timeinconsistencies, it is useful to contrast our findings with some central questions in the political-economy literature. We often see that restrictions on the set of policies that democratically elected governments can implement are viewed as welfare-improving; a prominent example is the European Union public deficit restrictions as stated in the Maastricht Treaty. On a theory level, Persson and Svensson (1989) show that without institutional constraints, time-inconsistent preferences lead the current government to exert control over its successors behavior by running deficits. Tabellini and Alessina (1990) argue that the lack of current majority's control over future voters most-preferred composition of spending tends to create current deficits, as a solution to the commitment problem. More directly related to our setting, Glazer (1987) finds that uncertainty of future voting outcomes biases current public investment towards durable long-term 
physical capital, and, more normatively, Bassetto and Sargent (2006) argue in favor of the golden rule where physical long-term public investments should be exempted from deficit restrictions.

Our results share the positive tone of this literature, as the current public investments -in the absence of cost-benefit rules - are used to tie the hands of the future agents. However, on the normative side, we argue against simple behavioral rules eliminating discretion by the current decision maker as not welfare enhancing. The normative conclusion we reach is that such rules must be part of a larger package that not only corrects for distortions in the composition of temporal spending but also in the intertemporal choices (i.e., overall savings). Thus, while reasons differ, we concur with Tabellini and Alessina (1990): "There is a role for institutions that enable society to separate its intertemporal choices from decisions concerning the allocation of resources within any given period".

\section{Infinite horizon model}

\subsection{The setting}

Consider a sequence of periods $t \in\{1,2, \ldots\}$ where gradual public investments, denoted by $q_{t} \geq 0$, are made to build up a public-asset, denoted by $s_{t} \geq 0$. The public asset accumulates as a function of the existing stock $s_{t}$ and current investment $q_{t+1} \geq 0$ in the next-period asset:

$$
s_{t+1}=\varphi\left(s_{t}, q_{t+1}\right)
$$

where we assume that $\varphi($.$) is increasing, bounded, and twice continuously differentiable$ in its arguments. This formulation is general enough to allow for multiple interpretations. The model could be interepreted as a stylized model of education where the future human capital depends on investments and past levels of the capital, or we can think of $s_{t}$ as public infrastructure where the final service depends on the quality of current infrastructure determined by accumulated investments. Variable $s_{t}$ could alternatively be an index for biodiversity which is maintained by continual effort. In climate change,

$s_{t}$ can measure the reduction of the greenhouse-gas stock from a pre-determined level, and $q_{t+1}$ is the current abatement effort.

The budget accounting equations between the periods are equation (33) and

$$
c_{t}+k_{t+1}+q_{t+1}=f\left(k_{t}\right) .
$$


In each period, the representative consumer makes the consumption and investment decisions, and derives utility from its own consumption and the public good. The consumer's welfare is

$$
w_{t}=u\left(c_{t}\right)+v\left(s_{t}\right)+\rho \sum_{\tau=t+1}^{\infty} \theta^{\tau-t-1}\left[u_{\tau}\left(c_{\tau}\right)+v_{\tau}\left(s_{\tau}\right)\right]
$$

where we identify dynamically consistent preferences by $\rho=\theta$, so that each future period $\tau>t$ is discounted with the same discount factor $\theta^{\tau-t}$. The dynamically inconsistent preferences are identified by $\rho \neq \theta$, and this model lends itself to the interpretations suggested by Phelps and Pollak (1968), and Laibson (1997). ${ }^{16}$ In particular, $\rho<\theta$ is consistent with pure altruism towards later decision makers (see Saez-Marti and Weibull (2005)). We also allow for $\rho>\theta$; it will become clear shortly that "over-investment" in the public asset can also occur in this case.

In the equilibrium analysis below we confine attention to Cobb-Douglas production functions $f\left(k_{t}\right)=k_{t}^{\alpha}$ and $\varphi\left(s_{t}, q_{t+1}\right)=s_{t}^{\delta} q_{t+1}^{1-\delta}$, where $0<\alpha<1$ and $0<\delta<1,{ }^{17}$ and assume logarithmic utilities for consumption $u\left(c_{t}\right)=\ln \left(c_{t}\right)$ and for the public asset $v\left(s_{t}\right)=\omega \ln \left(s_{t}\right)$ where $\omega>0$. The incentive to deviate from the cost-benefit requirement will depend, in addition to the time-inconsistency parameters, on the relative persistence of the public asset, i.e., on how large is parameter $\delta$ in relation to $\alpha$ and $\omega$.

The equilibrium outcome depends on the restrictions made on the strategies available (see Krusell et al. 2002, and Karp 2007). To obtain a comparison with the consistent preferences case $(\rho=\theta)$, we impose the differentiability and symmetry restriction on the strategies, i.e., each generation is assumed to use the same pair of differentiable policy functions $k_{t+1}=g\left(k_{t}, s_{t}\right)$, and $q_{t+1}=h\left(k_{t}, s_{t}\right)$. Under these assumptions and the functional forms for production and utility, we can find equilibrium strategies where investments shares $1>g>0$ and $1>h>0$ are constant fractions of the output:

$$
\begin{aligned}
& k_{t+1}=g f\left(k_{t}\right) \\
& q_{t+1}=h f\left(k_{t}\right) .
\end{aligned}
$$

The stationarity of investment shares is well known for consistent preferences $(\rho=\theta)$ under this specification, and we will derive such policies explicitly also for inconsistent

\footnotetext{
${ }^{16}$ To obtain the $\beta, \delta$ model, define $\beta=\rho / \theta, \delta=\theta$ and indentify inconsistent prefrences by $\beta<1$. We want to indentify inconsistencies by $\rho \neq \theta$ to maintain an easy comparison with the three period model; we can even think of "short-sighted preferences", with $\rho>0, \theta=0$, where the current generation cares about the immediate future, but not about those in the future further away.

${ }^{17} \mathrm{We}$ follow the custom use of $\alpha$ for the capital-output elasticity. When using time subscripts, the $\alpha_{t}$ refer to welfare weights while $\alpha_{t}^{\prime}$ refer to utility weights.
} 
preferences $(\rho \neq \theta) .{ }^{18}$ Since all policies in the remainder of this paper take the form (36)(37), we can make some useful conclusions for this class of policies before the equilibrium analysis in Section 3.2.

Given (35), we can express the equilibrium welfare as

$$
w_{t}=u\left(c_{t}\right)+v\left(s_{t}\right)+\rho V\left(k_{t+1}, s_{t+1}\right),
$$

where the (auxiliary) value function satisfies

$$
V\left(k_{t}, s_{t}\right)=u\left((1-g-h) f\left(k_{t}\right)\right)+v\left(s_{t}\right)+\theta V\left(g f\left(k_{t}\right), \varphi\left(s_{t}, h f\left(k_{t}\right)\right)\right) .
$$

We derive in the Appendix ${ }^{19}$ the parametric form for the value function, applying to all equilibria considered in this paper:

Lemma 3 The value function implied by policies (36)-(37) has the following parametric form

$$
V\left(k_{t+1}, s_{t+1}\right)=\xi \ln \left(k_{t+1}\right)+\frac{\zeta}{1-\delta} \ln \left(s_{t+1}\right)+\theta \mu[\xi \ln (g)+\zeta \ln (h)]+\mu \ln (1-g-h)
$$

where $\xi, \zeta, \mu>0$ are parameters independent of $\rho$.

The Lemma is very useful as it immediately establishes some important features of any equilibrium with constant investment shares. Notice that $g$ and $h$ in the value function refer to the future investment shares, from period $t+1$ onwards. The variables $k_{t+1}$ and $s_{t+1}$ are the current choices. There is no interaction between $g, h$, and $k_{t+1}, s_{t+1}$, so that the current optimal choices of $k_{t+1}$ and $s_{t+1}$ are independent of future investment shares $g$ and $h$. As $\ln \left(s_{t+1}\right)=\delta \ln \left(s_{t}\right)+(1-\delta) \ln \left(q_{t+1}\right)$, and one unit of investment in $k_{t+1}$ should yield the same marginal value as one unit investment in $q_{t+1}$, the Lemma shows that the current investment ratio (maximizing $w_{t}$ in (38)) is a constant given by $k_{t+1} / q_{t+1}=\xi / \zeta$ which is independent of the short-term time-preference parameter $\rho$ (see Appendix for the expressions of the parameters $\xi$ and $\zeta$ ). The shares $g^{*}$ and $h^{*}$ maximizing the value function also satisfy $g^{*} / h^{*}=\xi / \zeta$, implying that current investment shares line up with a time-consistent planner with time-discount factor equal to the agent's long-term factor $\theta$. The short-run time preference $\rho$ determines the overall savings, but the division between investment opportunities is determined by the long-run preference $\theta$.

\footnotetext{
${ }^{18}$ We do not consider non-linear symmetric stationary strategies; on that, see Karp (2007). Moreover, there could be equilibria in symmetric but non-stationary strategies.

${ }^{19}$ All proofs not in the text are in the Appendix.
} 
The cost-benefit rule equates the utility-weighted returns on investments in $k$ and $s$, i.e., the cost-benefit ratio (CBR) equals unity. In Appendix we derive this condition generally and show that it can be expressed for any $(g, h)$-policy as follows:

Remark 2 The infinite-horizon cost-benefit rule for policies of the form (36)-(37) is

$$
1=\frac{h(\alpha-\delta g)}{g \omega(1-\delta)(1-g-h)}=C B R .
$$

Pareto efficient investment shares $g$ and $h$ must satisfy rule (39), but the rule can also hold for some other $(g, h)$-policy, as we will see shortly. In this infinite-horizon setting, a Planner-equivalent outcome is, as in Barro (1999), an allocation that would be optimal for some consistent-preference decision-maker. Such an allocation must satisfy (39) and it must also imply a geometric discount factor that, if applied, would justify the choices for the fictitious Planner. Given the allocation, the "equilibrium discount factor" can be found from the Euler equation for consumption:

$$
\gamma \equiv \frac{u_{t}^{\prime}}{u_{t+1}^{\prime} R_{t, t+1}}=\frac{c_{t+1}}{c_{t} R_{t, t+1}}=\frac{c_{t+1}}{c_{t}} \frac{g}{\alpha} \frac{k_{t+1}}{k_{t+2}}=\frac{g}{\alpha}
$$

where $R_{t, t+1}$ is the compound rate for capital and the last step uses $c_{t} / k_{t+1}=(1-g-h) / g$.

When $\gamma<1$, we can view $\gamma$ as the discount factor for the Planner choosing the allocation, so that $\gamma^{t-1}$ are the Planner's utility weights for periods $t \geq 1$. More precisely, we can state:

Lemma 4 An equilibrium with $\gamma=g / \alpha<1$ is Planner-equivalent if and only if the cost-benefit rule (39) holds.

When observing a constant investment share equilibrium satisfying the cost-benefit rule, the result implies that there is a Planner associated with it, but how to verify if the Planner is representative, i.e., chooses a Pareto efficient outcome? The answer turns out to be simple:

Lemma 5 The Planner with $\gamma=g / \alpha<1$ is representative if and only if $\gamma \geq \max \{\rho, \theta\}$.

In the Appendix, we use the lower bound on the "equilibrium discount factor" $\gamma$, i.e. the condition $\gamma \geq \max \{\rho, \theta\}$, to show that the welfare weights remain positive, and also that only in this case such weights can be found. Intuitively, $\gamma$ can be seen as the discount factor that makes the first generation look like a consistent-preferences Planner; when this Planner puts a per-period weight factor larger than $\rho$ and $\theta$ on each generation's 
utility, then the implied equilibrium utility weights are large enough to leave room for positive welfare weights. It is not obvious whether this can hold in equilibrium - in particular so when the cost-benefit requirement is imposed as a rule of the game.

\subsection{Equilibrium}

Given the background from the previous section, it is now straightforward to assess the efficiency properties of the equilibrium. Considering the symmetric equilibrium where each period representative consumer chooses the same pair $(g, h)$, we can readily see the continuation value for each investment level from Lemma 3, and determine the equilibrium investment shares $g$ and $h$ from the first-order conditions for $k_{t+1}$ and $q_{t+1}$,

$$
\begin{aligned}
& u^{\prime}\left(c_{t}\right)=\rho V_{k}\left(k_{t+1}, s_{t+1}\right), \\
& u^{\prime}\left(c_{t}\right)=\rho \varphi_{q_{t+1}} V_{s}\left(k_{t+1}, s_{t+1}\right) .
\end{aligned}
$$

Given the functional form from Lemma 3, the equilibrium best-responses (41) and (42) can be written as

$$
\begin{aligned}
k_{t+1} & =\rho \xi c_{t} \\
q_{t+1} & =\rho \zeta c_{t} .
\end{aligned}
$$

Using $k_{t+1} / q_{t+1}=g / h$ and $c_{t} / k_{t+1}=(1-g-h) / g$ together with (43)-(44), we can express the equilibrium policies as follows (using the expressions for $\xi$ and $\zeta$ in Appendix):

$$
\begin{aligned}
g & =\frac{\rho \xi}{1+\rho \zeta+\rho \xi} \\
& =\rho \alpha \frac{1-\delta \theta+\theta \omega(1-\delta)}{1-\delta \theta+\rho \omega(1-\delta)+\alpha(1-\delta \theta)(\rho-\theta)}, \\
h & =\frac{\rho \zeta}{1+\rho \zeta+\rho \xi} \\
& =\rho \omega \frac{(1-\alpha \theta)(1-\delta)}{1-\delta \theta+\rho \omega(1-\delta)+\alpha(1-\delta \theta)(\rho-\theta)} .
\end{aligned}
$$

We see that when preferences are time-consistent $(\theta=\rho)$, the equilibrium investment in $k$ has the familiar form $g=\alpha \rho$, and the equilibrium discount factor is, as it should, $\gamma=$ $\frac{g}{\alpha}=\rho<1$. When preferences are time-inconsistent $(\theta \neq \rho)$, we can obtain the intuitive result that the equilibrium discount factor is between the two conceivable extremes:

Lemma 6 For all $\rho \neq \theta$, the equilibrium policy $g$ satisfies

$$
\min \{\rho, \theta\}<\gamma=\frac{g}{\alpha}<\max \{\rho, \theta\}<1 .
$$


The reasoning for this result (formally proved in the Appendix) is straightforward. The current agent cares more for total future welfare, and thus saves more, than a representative planner who would have consistent preferences with discount factor satisfying $\gamma=\min \{\rho, \theta\}$. At the same time, the current agent cares less, and saves less, compared to a representative planner who would have consistent preferences with $\gamma=\max \{\rho, \theta\}$. Clearly, the equilibrium savings must be somewhere between the extremes.

We can now describe the equilibrium outcome as depending on the inconsistency of the preferences and the relative persistency of the public asset. For ease of exposition, we use $C B R$ as a shorthand for the cost-benefit ratio, expressed on the right-hand side of the cost-benefit rule (39). We plug in the equilibrium policies (45) and (46) to (39) to obtain:

$$
C B R=1+\frac{(\theta-\rho)(\delta(1+\omega)-\alpha-\omega)}{(1-\theta \delta)+(1-\delta) \theta \omega}
$$

This is a closed form expression for the equilibrium cost-benefit ratio, implying:

Proposition 3 Returns on public investments fall short of returns on capital $(C B R>1)$ in equilibrium if and only if $(\theta-\rho)\left(\delta-\frac{\alpha+\omega}{1+\omega}\right)>0$. The equilibrium is Planner-equivalent if and only if either (i) $\theta=\rho$, or (ii) $\delta=\frac{\alpha+\omega}{1+\omega}$.

The proof is a matter of straightforward verification. The latter part follows by the equivalence of the Planner and the cost-benefit rule $(C B R=1)$ that we explicated in Lemma 4. While the equilibrium deviation from the cost-benefit rule is not surprising given our arguments from three periods, the result gives more structure to the determinants of the deviation. In particular, since the commitment provided by the public asset depends on its persistence relative to the traditional capital, the degree of over- or underinvestment depends not only on preferences but also on persistence. A large long-term discount factor $(\theta>\rho)$ was previously shown to be a reason for over-investment (i.e., costs exceeding benefits, $C B R>1$ ), but now the public asset should also be persistent enough to satisfy $\delta>\frac{\alpha+\omega}{1+\omega}$. Otherwise, the agent will under-invest in the public asset.

When preferences are time-consistent $(\theta=\rho)$, the cost-benefit rule will hold and, of course, it is possible to associate a Planner with the allocation, i.e., we can think that the dynastic first-period agent is the Planner. But this outcome also arises when the persistence of the public asset exactly matches the persistence of welfare transferred to future generations through capital $\left(\delta=\frac{\alpha+\omega}{1+\omega}\right)$, i.e., the equilibrium can be interpreted as a Planner's allocation, irrespective of the structure of time preferences $(\rho=\theta$, and $\rho \neq \theta$ ). This result sheds light on the generality of the observational equivalence between 
the equilibrium outcome and that obtained under consistent preferences, pointed out by Barro (1999). With more than one capital good, the observational equivalence follows only in the knife-edge case identified here.

The Planner-equivalence does not imply welfare efficiency, however. Lemma 6 implies that the exponential decrease in utility weights $\gamma$ associated with the equilibrium is too large.

Proposition 4 Suppose preferences are inconsistent, $\theta \neq \rho$, but $\delta=\frac{\alpha+\omega}{1+\omega}$ so that the equilibrium is Planner-equivalent. This Planner is not representative, i.e., Pareto efficiency is not achieved.

Efficiency requires $\gamma \geq \max \{\rho, \theta\}$ but this contradicts Lemma 6 above. The result thus implies that the equilibrium can never reach Pareto efficiency when preferences are dynamically inconsistent. This result is not surprising; while the equilibrium satisfies temporal efficiency in the sense that the composition of investments is optimal, the overall savings still deviate from the efficient savings for the reasons known from the one capital-good Ramsey saving problems with hyperbolic preferences.

\subsection{Cost-benefit law equilibrium}

We consider now whether the cost-benefit law, similar to that studied in three-periods, can improve welfare. We assume that the cost-benefit requirement is an institutional constraint dictating that all public investments must earn the same return as capital investments. As in three periods, we may think that the requirement is implemented administratively, e.g., through a budget office scrutinizing the investment plan at the end of each period. Other than this per-period check on the composition of spending, each generation is free to choose, within the resource constraints, the overall level of investment and consumption. With infinite horizon, the welfare implications of the costbenefit law are less obvious than in three periods, as the current generation can potentially benefit from the future generations' adherence to the law - in three periods we could not address the full dynamic potential of the cost-benefit law, as it was only binding for the first generation by construction.

Formally, we consider a game where each generation chooses investments $k_{t+1}$ and $q_{t+1}$ subject to the constraint that the cost-benefit ratio must equal unity $(C B R=1)$, and the restriction on strategies that each future generation applies a constant investment share policy. We can think of each period involving two steps. In the first, the agent 
decides only on the overall investment $I_{t+1}=k_{t+1}+q_{t+1}$ and, in the second, the amount $I_{t+1}$ is divided between the two purposes such that $C B R=1$ is satisfied, understanding that each future generation will follow the same procedure.

Since we are focusing on the constant investment share policies, and the cost-benefit rule (39) was derived for any such policy, we can solve for the investment shares from the cost-benefit rule (39):

$$
\frac{q_{t+1}}{k_{t+1}}=\frac{\omega(1-\delta)(1-g-h)}{\alpha-\delta g} \equiv \frac{\eta}{1-\eta}
$$

The left-hand side refers to current investment decisions, and the right hand side refers to future investment decisions that are considered as given by the future agents' strategies in the subgame-perfect equilibrium. By definition, $\eta$ is the share of the public asset investment in total investments $I_{t+1}$. Given the future policies, we only need to consider the best-response today for total savings $I_{t+1}$, as the shares follow by $k_{t+1}=(1-\eta) I_{t+1}$, and $q_{t+1}=\eta I_{t+1}$. We must thus have

$$
\frac{d w_{t}}{d I_{t+1}}=-\frac{d u_{t}}{d c_{t}}+\rho\left((1-\eta) \frac{d V_{t+1}}{d k_{t+1}}+\eta \frac{d V_{t+1}}{d q_{t+1}}\right)=0
$$

We have derived the form for the value function for any pair of $(g, h)$-policies, so we can readily assess the implications of the cost-benefit rule on total savings:

Remark 3 The cost-benefit law does not change total investments, but only the shares of capital and the public good. Investment in the public good decreases if and only if $(\theta-\rho)\left(\delta-\frac{\alpha+\omega}{1+\omega}\right)>0$

Formally, we can see the first part of the result from the first-order condition (47) which, given Lemma 3, can be restated as

$$
I_{t+1}=\rho(\xi+\zeta) c_{t}
$$

or, by $I_{t+1}=\frac{g+h}{1-g-h} c_{t}$,

$$
g+h=\frac{\rho \xi+\rho \zeta}{1+\rho \zeta+\rho \xi} .
$$

which equals the equilibrium total savings implied by (45) and (46). This result is already indicative of the fact that the cost-benefit requirement alone cannot deliver a Pareto efficient outcome, as it does not correct for the distortions in overall savings. For the second part, note that in this equilibrium we must have $C B R=1$ so that, if $C B R>1$, the public-asset investment share declines when compared to the equilibrium without the 
cost-benefit rule, see Proposition $3 .^{20}$ Thus, the cost-benefit law either pulls resources away from public investment or towards it, depending on the relative persistence of the public asset and preference inconsistencies.

The cost-benefit law restores productive efficiency in the sense that all assets earn seemingly appropriate returns, so that by observing such an outcome we might conclude that efficiency has been achieved. However, in levels the outcome is inefficient due to the fact that there are distortions in savings, when preferences are inconsistent:

Proposition 5 If $\rho \neq \theta$, the cost-benefit law equilibrium is not Pareto efficient.

We can verify the result by noting that the law implements a Planner-equivalent outcome with discount factor $\gamma<1$. Such an economy grows by investing fraction $g=\alpha \gamma$ of the output in capital $k$. The equilibrium first-order condition for capital investment implies a constant investment-consumption ratio:

$$
\frac{k_{t+1}}{c_{t}}=\rho \alpha\left[\frac{1}{(1-\alpha \theta)}+\frac{(1-\delta) \omega \theta}{(1-\alpha \theta)(1-\delta \theta)}\right] .
$$

Since observationally equivalent Planner's outcome must satisfy the same ratio, we have

$$
\rho \alpha\left[\frac{1}{(1-\alpha \theta)}+\frac{(1-\delta) \omega \theta}{(1-\alpha \theta)(1-\delta \theta)}\right]=\gamma \alpha\left[\frac{1}{(1-\alpha \gamma)}+\frac{(1-\delta) \omega \gamma}{(1-\alpha \gamma)(1-\delta \gamma)}\right],
$$

where the right-hand side is the Planner's version of the ratio. However, if $\gamma \geq \max \{\rho, \theta\}$, the equation cannot hold (the right-hand side is larger). Thus, we must have $\gamma<$ $\max \{\rho, \theta\}$ and, by Lemma 5, Pareto efficiency is not achieved, so the Planner is not representative.

While the cost-benefit law does not restore full efficiency, it might be argued that the productive inefficiency removed produces at least a Pareto improvement. However, not even this can be achieved:

Proposition 6 The implementation of the cost-benefit law from period $t$ onwards implies a welfare loss for generation $t$, compared to the equilibrium without the law.

The result shows that the three period conclusion extends to the infinite horizon: the first generation under the law cannot sufficiently benefit from the later generations' adherence to the law. Yet, the cost-benefit rule may create some overall economic surplus in the future, that could be used to support more complicated behavioral strategies for

\footnotetext{
${ }^{20}$ The cost-benefit ratio in (39) strictly decreases in $h$ when $g+h$ remains constant, as is the case in this comparison.
} 
distributing the surplus, thereby supporting the rule as an equilibrium outcome without imposing it as an institutional constraint. For this reason, we study whether there are efficiency gains in the long-run. In the Appendix we provide the proof for the next proposition, which show that there is no long-run efficiency gain: welfare strictly decreases if preferences are quasi-hyperbolic.

Proposition 7 The cost-benefit law decreases the steady-state welfare if $\theta>\rho$.

The illustration after the next section provides a quantitative assessment of the steady state losses as well as gains from focusing on savings-based rules that we consider next.

\subsection{Efficient saving rules}

The cost-benefit rule generates no social value because it does not solve the source of the allocation problem, namely the distortion in the inter-generational income transfer. Savings rules, which directly target income transfers, might be more effective in achieving efficiency gains. Indeed, we find that if distortions in savings can be corrected, the costbenefit rule will be self-fulfilling, i.e., there is no need to make it institutionalized. This observation corroborates the conclusion that the "cost-benefit commitment" as such has no social value. To illustrate, assume now altruistic (i.e., quasi-hyperbolic) preferences, $\theta>\rho$, and consider Pareto efficiency which requires Planner-equivalence and also that the Planner's equilibrium discount factor, denoted by $\gamma$ above, is at least equal to $\theta$ (see Lemma 5). This tells us that minimal Pareto-efficient savings satisfy $g=\alpha \gamma=\alpha \theta$. To determine the Pareto efficient investments in the public asset, we consider the investment plans (45) and (46) and adjust these so that they are in line with a representative Planner with discount factor $\theta$, that is, we substitute $\theta$ for $\rho$ everywhere. This gives $g=\alpha \theta$, and $h / g=\zeta / \xi$. Minimal Pareto-efficient savings thus require: ${ }^{21}$

$$
g+h=\alpha \theta\left(1+\frac{\zeta}{\xi}\right)
$$

If such a policy on overall savings $g+h$ could be written into a law, then all generations would voluntarily choose to follow the cost-benefit rule: the socially optimal division of savings for private and public uses lines up with preferences of each generation (see the discussion below Lemma 3). The result underscores the fundamental nature of the long-term cost-benefit problem: when savings are socially optimal, decision-makers have

\footnotetext{
${ }^{21}$ Notice that if savings are set above the minimal value, so that $\gamma>\theta$, then the associated investment shares for private and public capital do not equal $\xi$ and $\zeta$.
} 
no need to look for commitment devices, and the investment biases disappear. However, Pareto efficiency may not be self-enforcing, i.e., it need not be in the interest of any generation to set a law that implements Pareto efficiency if their own welfare decreases. Let us now consider savings-investment rules that are self-enforcing. Let the first generation propose an investment rule $(\widehat{g}, \widehat{h})$ that would serve its own interests, understanding that the rule needs to satisfy all future generations' incentive constraints, i.e., they would not benefit from deviating to the benchmark equilibrium where no rules apply. Consider the welfare of generation $t$ depending on the assets received $\left(k_{t}, s_{t}\right)$ and investment shares $(g, h)$ that are constants from period $t$ onwards. Substituting the value function in Lemma 3 to the welfare in (38), we find welfare $w_{t}$ for generation $t$ as a function of investment shares $(g, h)$. Taking derivatives, optimal investment shares are calculated as:

$$
\begin{aligned}
\widehat{g} & =\frac{\rho \xi}{1+\rho-\theta+\rho \xi+\rho \zeta} \\
\widehat{h} & =\frac{\rho \zeta}{1+\rho-\theta+\rho \xi+\rho \zeta} .
\end{aligned}
$$

Note that these are just the previous SPE shares with an additional term in the denominator, $\rho-\theta<0$, so that investments in both assets increase; we find the privately optimal investment shares to lie between the benchmark equilibrium shares and the Pareto-efficient shares (inequalities mirror when $\theta<\rho$ ): ${ }^{22}$

$$
g^{B A U}<\widehat{g}<g^{P E} \text { and } h^{B A U}<\widehat{h}<h^{P E} \text { iff } \theta>\rho .
$$

Similar to the Pareto efficient investment shares, the investment ratio between the two assets $\widehat{g} / \widehat{h}$ is consistent with private preferences of each generation, so that the rule only needs to specify total savings $\widehat{g}+\widehat{h}$, and it can leave the division over investments to each generation's discretion. Moreover, the rule optimizes welfare of generation $t$, but as the same rule optimizes welfare for all future generations, subsequently, no generation benefits from deviation, understanding that a deviation triggers a fall of future savings back to the benchmark levels.

The self-enforcing savings rule is clearly a Pareto improvement but yet it does not imply full satisfaction of the cost-benefit rule - as the total savings still fall short of the first best, the commitment value of the long-term asset is used also in this equibrium. To assess the deviation, we plug the equilibrium policies (49)-(50) to the right-hand

$$
{ }^{22} \widehat{g}<g^{P E} \text { follows from } g^{P E}=\frac{\theta \xi}{1+\theta \xi+\theta \zeta}
$$


side of the cost-benefit rule (39) to obtain the following expression for the self-enforcing cost-benefit ratio:

$$
\widehat{C B R}=1+\frac{(\theta-\rho)(\delta(1+\omega)-\alpha-\omega)}{(1-\theta \delta)+(1-\delta) \theta \omega}\left(\frac{1-\theta}{1+\rho-\theta}\right) .
$$

The description of Proposition 3 applies also in this equilibrium but, for given parameters, the departure from the efficient cost-benefit ratio $(C B R=1)$ is reduced, as $(1-\theta) /(1+$ $\rho-\theta)<1$. This reflects again the fact that savings rules reduce the need for using long-term assets as commitment. This effect will show clearly in the illustration.

\subsection{Illustration: climate policies}

To see whether some ballpark numbers can make the losses from the cost-benefit law visible as well as the potential gains from focusing on savings directly, we carry out a simple exercise where we consider the climate system as public asset. While the formulation is too simple to capture the dynamics of the climate problem, we nevertheless believe the illustration delivers an important and novel insight on the "climate-policy ramp" discussion that followed the Stern Review (2006), i.e., whether an initially moderate reduction effort is optimal followed by a gradual increase in efforts when the global carbon stock increases, or whether we should aim for immediate and high emission reduction targets. At the heart of the debate is the choice of the discount rate that depends primarily on the rate of pure time-preference and on the consumption-smoothing preferences assumed (see Nordhaus 2007, and Weitzman 2007). ${ }^{23}$ Assuming the same parametric forms as in the above analysis, we use the structure to interpret the main opposing views in the "climate-policy ramp" discussion, and then proceed to the illustration of the cost-benefit law and savings-rule equilibria; these two policy cases match the opposing views remarkably well. ${ }^{24}$

We thus consider the case where one must choose between investments in capital or in a very durable public asset (the climate system). Assuming time steps of 20 years we may treat the neoclassical capital as a broad man-made stock, possibly including human capital, that is fully depreciated in one period. We set the output-capital elasticity equal

\footnotetext{
${ }^{23}$ To be sure, there is also disagreement as to the development of emission reduction costs. Some believe deep emission cuts should wait until costs have come down, while others assert that cost reductions only happen after we start reducing emissions.

${ }^{24}$ We notice that the parametric form of our model implies constant investment shares, so that shortrun and long-run policies are symmetric, but the dynamic structure of the capital and public assets build up will cause substantial differences between short and long-term effects.
} 
to $\alpha=0.5$. The public asset represents the accumulated reduction of the atmospheric greenhouse-gas stock relative to a benchmark (business as usual) path in which no efforts are undertaken to reduce greenhouse-gas emissions. The climate systems evolves slowly; the uptake of antropogenic emissions implies that atmospheric $\mathrm{CO}_{2}$ particles depreciate approximately at 0.5 per cent per year. The persistence of the public asset of $99.5 \%$ per year implies $\delta=0.9$. Let us assume that the true preferences are such that the agents discount short-term utility at an annual rate of 2.5 per cent, implying $\rho=0.5$, but they do not differentiate much between periods beyond the first 20 years; we set $\theta=0.95$.

The business as usual (BAU) scenario is the one where the markets for private capital work well, but the public good is undersupplied. As we think of our public-good stock as the difference between the BAU and actual equilibrium greenhouse-gas stocks, the BAU public-good stock is zero. The BAU equilibrium is then defined by setting $\omega=0$, giving $g=0.323$ (see eq. (45)), roughly consistent with the historical empirical data on gross investment rates (Mankiw et al. 1992). We now develop two simple policy experiments based on this BAU benchmark, following the approach that has been associated with Nordhaus' and Stern's previous studies, respectively. In the first experiment, which we label "positive discounting", ${ }^{25}$ we interpret the historically observed BAU equilibrium as a time-consistent preferences equilibrium (see also Barro (1999) for the observational equivalence), and, by presuming $\rho=\theta=\gamma$, determine the time preference factor by our version of the Ramsey savings-rule, (40) as $\gamma=g / \alpha=0.658$. The pure time discount factor is equivalent to an annual rate of pure time preference of $.658^{-0.05}-1=0.021$, broadly in line with Nordhaus (2007). According to these assumptions, the optimal policy does not imply changes in investments in the capital stock, i.e. $g=0.323$, while public policy will set investments in the public asset at a level $h=0.010$, if we assume $\omega=0.1$ (we keep $\omega$ set at this level from now on). That is, according to this interpretation of preferences, 1 per cent of global income should be used to preserve the environment, i.e., to reduce the atmospheric greenhouse-gas stock.

In the "prescriptive discounting" experiment, the argument is that the historically observed savings behavior does not reveal our true long-term preferences, which rather should be approximated by the discount factor $\theta=0.95 .^{26}$ As shown in Table 1 , gross

\footnotetext{
${ }^{25}$ We use the terms positive and prescriptive as common in this literature. In our context, employing the time-inconsistent preference structure could also be considered a positive approach to discounting.

${ }^{26}$ It is not necessary that the discount rate based on ethical reasoning coincides with the discount rate that the individuals apply for their own long-term preferences. But if ethical discounting has to be supported by individuals in society, it seems a reasonable assumption.
} 


\begin{tabular}{cccc}
\hline \hline variable & BAU & positive discounting & prescriptive discounting \\
\hline$g$ & .323 & .323 & .475 \\
$h$ & 0 & .010 & .032 \\
$\gamma$ & .658 & .658 & .95 \\
\hline
\end{tabular}

Table 1: Investment shares $(g, h)$ and equilibrium discount factor $(\gamma)$, for the business as usual $(B A U)$, "positive discounting", and "prescriptive" equilibria, for parameters $\alpha=.5, \omega=.1, \delta=.9, \rho=.5, \theta=.95$.

savings in capital jump to $g=.475$ and the public-good investments about triple to $h=.032$. The discount factor is, of course, $\theta=\gamma=0.95$ corresponding to an annual rate of pure time preference of 0.0025 . We notice that such a design for public policy is in the Pareto set for the true preferences (see Section 3.4), but it may be argued that the outcome is not realistic; a gross investment rate above 50 per cent cannot be supported by preferences with short-term annual discount rate of 2.5 per cent, a criticism often presented against Stern's (2006) choice of parameters. ${ }^{27}$

Three alternatives to the "positive discounting" and "prescriptive discounting" experiments become clear from our analysis, and these are reported in Table 2 for the same set of parameters. One policy design is based on the subgame-perfect equilibrium (SPE) where the planner is aware of the time-inconsistency problems implied by the preferences, and chooses optimal investments both in capital and greenhouse-gas stock reductions that maximize welfare taking into account the anticipated future policy choices. We find the SPE investment share in capital to remain almost in the same order as under positive discounting, $g=0.329$, but the public-good investment share doubles to the level $h=0.022$. The equilibrium policy leaves capital investments almost unchanged, but substantially increases climate investments as preference-inconsistencies motivate the use of the persistent climate asset for channeling wealth to the future. Consequently, and consistent with our analytical results, the cost-benefit ratio is over 2, implying that each dollar invested in climate mitigation yields a net present value benefit of less than half a dollar.

As the SPE investments in the public good are so far off the efficient cost-benefit ratio, it is natural to consider next the policy scenario that imposes the cost-benefit requirement. Consider thus implementing the cost-benefit law, as we have done throughout the analysis of this paper, and see Table 2 again. This policy diverts part of the resources

\footnotetext{
${ }^{27}$ See Dasgupta (2008) for a succinct elaboration of this criticism.
} 


\begin{tabular}{cccc}
\hline \hline variable & benchmark SPE & cost-benefit law & self-enforcing savings \\
\hline$g$ & .329 & .340 & .465 \\
$h$ & .022 & .011 & .032 \\
$\gamma$ & .658 & .680 & .929 \\
$C B R$ & 2.14 & 1 & 1.10 \\
$E V^{S S}$ & 0 & $-3.1 \%$ & $17.5 \%$ \\
\hline
\end{tabular}

Table 2: Investment shares $(g, h)$, equilibrium discount factor $(\gamma)$, the cost-benefit ratio $(C B R)$, and the steady-state equivalent variation $\left(E V^{S S}\right)$, for the benchmark subgameperfect, cost-benefit law, and self-enforcing savings equilibrium for parameters $\alpha=.5$, $\omega=.1, \delta=.9, \rho=.5, \theta=.95$.

from the public asset towards the capital stock, and the equilibrium investment in capital increases somewhat while the investment share of the public good returns to about the same level as in the positive discounting experiment, $h=0.011$. This reflects the idea that high capital opportunity costs renders immediate large investments in the climate asset unfavorable, resembling the "gradualism" approach advocated by Nordhaus.

The final policy scenario, in the last column of the Table 2, presents the selfenforcing savings rule. We see jump-start investments in the public good, $h=0.032$, but investments in the traditional capital increase as well. As the public planner can lineup present and future gross savings as a result of the savings rule, the optimal level of overall savings implies a modest .36 per cent annual pure rate of discount. Importantly, since both assets increase, the ex post cost-benefit ratio nears one. The self-enforcing equilibrium is observationally close to the prescriptive discounting experiment. Both policies have noticeable accumulative implications on the equilibrium paths, which we consider next.

Figure 1 provides a vivid illustration of the differences between the equilibria over time and of the implications for the "policy-ramp". For illustration of the transitions, the paths start with capital and public assets at 10 per cent of the SPE steady state level. It takes about 5 periods of 20 years for the capital stock to converge to its longrun level. The public asset takes much longer to converge, consistent with the climate change literature. A comparison between the solid- (SPE) and dotted-line (CBR) paths shows what the simple cost-benefit rule does: it shifts resources from the public-good stock $s$ (the reduction of the atmospheric carbon stock) towards the traditional capital $k$. This reflects the notion that private assets offer a better opportunity to contribute 

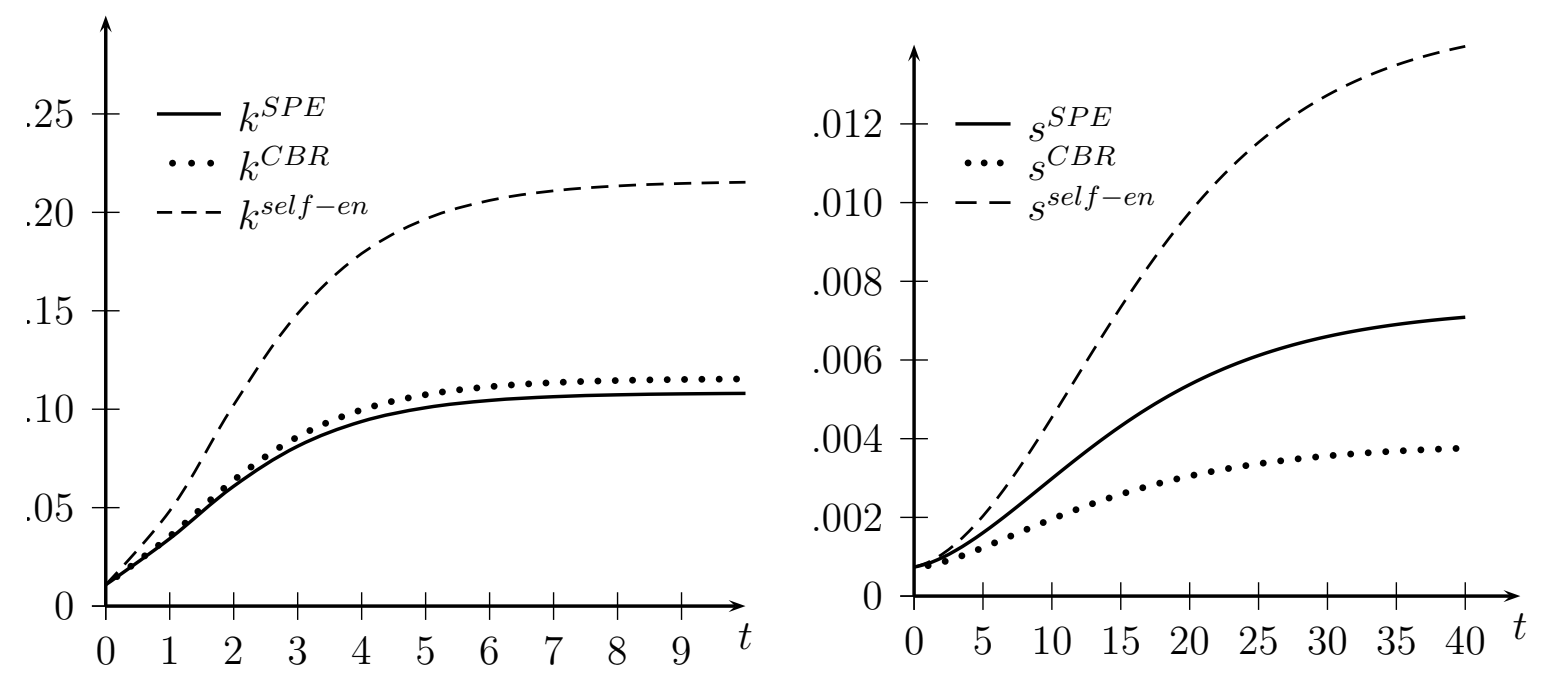

Figure 1: Left: Capital accumulation paths for the benchmark subgame-perfect $\left(k^{S P E}\right)$, cost-benefit law $\left(k^{C B R}\right)$, and self-enforcing savings-rule equilibrium $\left(k^{\text {self-en }}\right)$. Right: Corresponding public-good stocks. Unit of time is 20 years. Parameters from Table 1.

to future welfare compared to public assets, when costs of public investments exceed the net present value benefits. But welfare is lower under the cost-benefit law equilibrium for all generations (see Proposition 6 for the first generation, and Proposition 7 for the long run). In this illustration, the change in welfare is equivalent to a drop of about 3 per cent of consumption (see the last line of Table 2). ${ }^{28}$ The dashed lines show that the assets are made complementary by the savings rule: both the capital and public assets increase relative to the other paths. In this sense, the jump-start climate policies need not come at the expense of the capital accumulation path. For all generations welfare is highest in the self-enforcing savings equilibrium, and in the long run is equivalent to a 17.5 per cent increase in consumption (Table 2). If an increase in aggregate savings is infeasible, then in welfare terms the SPE equilibrium performs substantially better compared to the cost-benefit rule equilibrium.

\footnotetext{
${ }^{28}$ The steady-state equivalent variation calculation is demonstrated in the proof of Proposition 7.
} 


\section{Concluding remarks}

Public investments are often extremely long term by nature. Due to the long time horizon and difficulties in evaluating the future benefits, they present a challenge to the traditional cost-benefit analysis. We introduced a different complication: if preferences are known to be time-inconsistent such that the future ranking of current public decisions will be different from that today, how should the principles of the CBA be altered? We found that the persistence of the effects of current decisions lead to incentives to deviate from the standard cost-benefit requirements. Almost by definition public investments provide commitment to current preferences, and it makes sense to use this commitment to overcome the inconsistencies in public decision making over time.

We found no normative reason to insist on the use of stand-alone cost-benefit rules when preferences are inconsistent: the overall welfare is not maximized under such rules. The cost-benefit analysis is based on a narrow concept of efficiency, and imposing the cost-benefit rule does not even imply a Pareto improvement, let alone achievement of welfare Pareto efficiency.

One extension of the current infinite-horizon model is a more detailed application to education, energy, or climate change, e.g., by using a numerical integrated assessment model (IAM) linking the economy and the climate development. Based on our results on the observational equivalence between consistent and inconsistent preferences equilibria, we can conjecture that a standard IAM solution can also be interpreted as an equilibrium resulting from inconsistent preferences with an enforced cost-benefit rule. One can then explore with little effort the welfare loss from pursuing the cost-benefit rules (typically justified by a consistent preference framework) if the true underlying preferences are in fact inconsistent.

On a theory level, a natural alternative formulation is one where the current government understands that the future preferences are likely to be different but is unsure in which way. Alternatively, one may want to consider more deeply the source of inconsistency in public decision making. For example, it is well known that aggregation over individual heterogenous discount factors leads to average discount rates that decline with the time horizon (Weitzman 2000, Gollier and Zeckhauser 2005). As such this is not a source inconsistency in decentralized economy with heterogenous but consistent agents (Lengwiler 2005). However, in public decision making one may be forced to aggregate over individuals such that inconsistencies arise. We leave these interesting questions open for future research. 


\section{Appendix}

\section{Value function: Lemma 3}

We proceed in the following steps. First, we show that there are parameters $\xi, \zeta, a_{g}, a_{h}, \mu$ such that the value function can be written as

$$
V_{t}=\xi \ln \left(k_{t}\right)+\frac{\zeta}{1-\delta} \ln \left(s_{t}\right)+a_{g} \ln (g)+a_{h} \ln (h)+\mu \ln (1-g-h) .
$$

Then we analyze how $a_{g}$ and $a_{h}$ relate to the other parameters.

Given stationary investment shares, we can fully calculate all forward capital and public good levels. We use tildes to denote log-variables. The stock dynamics can then be written recursively as

$$
\begin{aligned}
\widetilde{k}_{t+1} & =\widetilde{g}+\alpha \widetilde{k}_{t}, \\
\widetilde{s}_{t+1} & =\delta \widetilde{s}_{t}+(1-\delta)\left(\widetilde{h}+\alpha \widetilde{k}_{t}\right) .
\end{aligned}
$$

Substitution allows us to find the complete future path of stocks $k_{t}$ and $s_{t}$ as a function of initial stocks $k_{1}$ and $s_{1}$, and the policy functions:

$$
\begin{aligned}
& \widetilde{k}_{t+\tau}=\alpha^{\tau} \widetilde{k}_{t}+\frac{1-\alpha^{\tau}}{1-\alpha} \widetilde{g} \\
& \widetilde{s}_{t+\tau}=\delta^{\tau} \widetilde{s}_{t}+\left(1-\delta^{\tau}\right) \widetilde{h}+\alpha(1-\delta)\left[\widetilde{k}_{t} \sum_{i=0}^{\tau-1} \alpha^{i} \delta^{\tau-i-1}+\widetilde{g} \sum_{i=1}^{\tau-1} \frac{1-\alpha^{i}}{1-\alpha} \delta^{\tau-i-1}\right]
\end{aligned}
$$

We can write equation (53) as

$\widetilde{s}_{t+\tau}=\delta^{\tau} \widetilde{s}_{t}+\left(1-\delta^{\tau}\right) \widetilde{h}+\alpha(1-\delta)\left[\frac{\delta^{\tau}-\alpha^{\tau}}{\delta-\alpha} \widetilde{k}_{t}+\frac{\alpha}{1-\alpha}\left(1-\delta^{\tau-1}-\alpha(1-\delta) \frac{\delta^{\tau-1}-\alpha^{\tau-1}}{\delta-\alpha}\right] \widetilde{g}\right.$

Now, we observe that using logarithms denoted by a tilde, we have $u_{t}=\widetilde{c}_{t}=\ln (1-$ $g-h)+\alpha \widetilde{k}_{t}, v_{t}=\omega \widetilde{s}_{t}$, and by the definition of the value function

$$
\begin{aligned}
V_{t} & =\sum_{\tau=0}^{\infty} \theta^{\tau}\left(u_{t+\tau}+v_{t+\tau}\right) \\
& =\frac{1}{1-\theta} \ln (1-g-h)+\alpha \sum_{\tau=0}^{\infty} \theta^{\tau} \widetilde{k}_{t+\tau}+\omega \sum_{\tau=0}^{\infty} \theta^{\tau} \widetilde{s}_{t+\tau} .
\end{aligned}
$$

Looking at (52) and (53) implies that the value function has the general parametric form stated in (51). We find the following parameters by direct summing over terms: 


$$
\begin{aligned}
\xi & =\alpha \sum_{\tau=0}^{\infty}(\theta \alpha)^{\tau}+\omega \alpha \frac{1-\delta}{\delta-\alpha} \sum_{\tau=0}^{\infty}\left((\theta \delta)^{\tau}-(\theta \alpha)^{\tau}\right) \\
& =\frac{\alpha}{1-\alpha \theta}+\omega \alpha \frac{1-\delta}{\delta-\alpha}\left(\frac{1}{1-\theta \delta}-\frac{1}{1-\theta \alpha}\right) \\
& =\frac{\alpha}{1-\alpha \theta}+\omega \alpha \frac{\theta(1-\delta)}{(1-\theta \alpha)(1-\theta \delta)} \\
& =\alpha \frac{1+\omega \theta-\delta \theta-\omega \delta \theta}{(1-\alpha \theta)(1-\delta \theta)} \\
\zeta & =(1-\delta) \omega \sum_{\tau=0}^{\infty}(\delta \theta)^{\tau} \\
& =\frac{(1-\delta) \omega}{(1-\delta \theta)} \\
\mu & =\frac{1}{1-\theta} .
\end{aligned}
$$

We now want to determine $a_{g}$ and $a_{h}$. As above, we could directly calculate the coefficients by summing all terms over time, but we can also derive the coefficients by a more subtle reasoning. For time consistent preferences, $\rho=\theta$, we can calculate the investment shares $g^{*}=k_{t+1} / y_{t}$ and $h^{*}=q_{t+1} / y_{t}$ that maximize

$$
w_{t}=u_{t}\left(y_{t}-k_{t+1}-q_{t+1}\right)+\theta V\left(k_{t+1}, s_{t+1} ; g, h\right)
$$

which gives

$$
\begin{aligned}
g^{*} & =\frac{\theta \xi}{1+\theta \xi+\theta \zeta}, \\
h^{*} & =\frac{\theta \zeta}{1+\theta \xi+\theta \zeta} .
\end{aligned}
$$

These values must be the same as those we can calculate directly from maximizing $V$ :

$$
\begin{aligned}
g^{*} & =\frac{a_{g}}{\mu+a_{g}+a_{h}}, \\
h^{*} & =\frac{a_{h}}{\mu+a_{g}+a_{h}} .
\end{aligned}
$$

It follows directly that $a_{g}=\mu \theta \xi$ and $a_{h}=\mu \theta \zeta$.

\section{Remark 2}

Consider allocation $(\mathbf{c}, \mathbf{q}, \mathbf{k})=\left\{c_{t}, q_{t}, k_{t}\right\}_{t=1}^{\infty}$ that maximizes the utility aggregator

$$
U(\mathbf{c}, \mathbf{q}, \mathbf{k})=\sum_{t=1}^{\infty} \alpha_{t}^{\prime}\left[u\left(c_{t}\right)+v\left(s_{t}\right)\right]
$$


for some utility weights $\alpha_{t}^{\prime} \geq 0$, satisfying $\sum_{t=1}^{\infty} \alpha_{t}^{\prime}<\infty$. Let us use the short-hand notation $\varphi_{q, t+1}=\varphi_{q}\left(s_{t}, q_{t+1}\right)$ and $\varphi_{s, t+1}=\varphi_{s}\left(s_{t}, q_{t+1}\right)$, and let $R_{t, \tau}=f_{t+1}^{\prime} \cdot f_{t+2}^{\prime} \cdot \ldots \cdot f_{\tau}^{\prime}$ be the compound rate of return for $k$, and $J_{t+1, \tau}=\varphi_{s, t+1} \cdot \varphi_{s, t+2} \cdot \ldots \cdot \varphi_{s, \tau}$ the compound rate of return for the public asset $\left(J_{t+1, t+1} \equiv 1\right)$. We prove first the following result:

Remark 4 The infinite-horizon cost-benefit rule is

$$
1=\left[\varphi_{q, t+1} \sum_{\tau=t+1}^{\infty} \frac{J_{t+1, \tau}}{R_{t, \tau}} \frac{v_{\tau}^{\prime}}{u_{\tau}^{\prime}}\right]^{-1}
$$

Proof. Capital investment $k_{t+1}>0$ satisfies

$$
\alpha_{t}^{\prime} u_{t}^{\prime}=\alpha_{t+1}^{\prime} u_{t+1}^{\prime} f_{t+1}^{\prime}
$$

This conditions holds between any two periods with positive investment, implying that for any $\tau \geq t+1$

$$
\alpha_{t}^{\prime} u_{t}^{\prime}=\alpha_{\tau}^{\prime} u_{\tau}^{\prime} R_{t, \tau}
$$

where $R_{t, \tau}=f_{t+1}^{\prime} \cdot f_{t+2}^{\prime} \cdot \ldots \cdot f_{\tau}^{\prime}$ is the compound rate of return for $k$. On the other hand, investment $q_{t+1}>0$ in the public asset satisfies

$$
\alpha_{t}^{\prime} u_{t}^{\prime}=\left[\alpha_{t+1}^{\prime} J_{t+1, t+1} v_{t+1}^{\prime}+\alpha_{t+2}^{\prime} J_{t+1, t+2} v_{t+2}^{\prime}+\ldots\right] \varphi_{q, t+1}
$$

Rearrange (55) to obtain

$$
1=\varphi_{q, t+1}\left[\frac{\alpha_{t+1}^{\prime} J_{t+1, t+1} v_{t+1}^{\prime}}{\alpha_{t}^{\prime} u_{t}^{\prime}}+\frac{\alpha_{t+2}^{\prime} J_{t+1, t+2} v_{t+2}^{\prime}}{\alpha_{t}^{\prime} u_{t}^{\prime}}+\ldots\right] .
$$

The expression on the right gives the benefit-cost ratio for a marginal increase in the public asset. The benefit from the investment $q$ is the increase in the next period public asset stock $\varphi_{q, t+1}$ times the sum of the utility-weighted compound returns $J_{t+1, \tau} v_{\tau}^{\prime}$ in periods $\tau \geq t+1$. The cost of the investment is the current utility loss which equals the return on capital investment $k$. To obtain an expression that does not depend on utility weights, we can replace $\alpha_{t}^{\prime} u_{t}^{\prime}$ with (56) to obtain expression (54). 
We obtain expression (39) by expanding the term in the brackets in (54),

$$
\begin{aligned}
\varphi_{q, t+1} \sum_{\tau=t+1}^{\infty} \frac{J_{t+1, \tau}}{R_{t, \tau}} \frac{v_{\tau}^{\prime}}{u_{\tau}^{\prime}} & =(1-\delta) \frac{s_{t+1}}{q_{t+1}} \sum_{\tau=t+1}^{\infty} \frac{\delta^{\tau-t-1} \frac{s_{\tau}}{s_{t+1}}}{g^{t-\tau} \alpha^{\tau-t} \frac{k_{\tau+1}}{k_{t+1}}} \frac{\omega c_{\tau}}{s_{\tau}} \\
& =(1-\delta) \frac{s_{t+1}}{q_{t+1}} \sum_{\tau=t+1}^{\infty} \frac{\delta^{\tau-t-1} \frac{s_{\tau}}{s_{t+1}}}{g^{t-\tau} \alpha^{\tau-t} \frac{k_{\tau+1}}{k_{t+1}}} \frac{(1-g-h) \omega k_{\tau+1}}{g s_{\tau}}(59) \\
& =(1-\delta) \frac{s_{t+1}}{q_{t+1}} \sum_{\tau=t+1}^{\infty} \frac{\delta^{\tau-t-1}}{g^{t-\tau} \alpha^{\tau-t}} \frac{(1-g-h) \omega k_{t+1}}{g s_{t+1}} \\
& =\frac{g}{h} \omega(1-\delta)(1-g-h) \sum_{\tau=t+1}^{\infty} \frac{\delta^{\tau-t-1} g^{\tau-t}}{\alpha^{\tau-t}} \\
& =\frac{g}{h} \frac{\omega(1-\delta)(1-g-h)}{\alpha} \sum_{\tau=0}^{\infty}\left(\frac{\delta g}{\alpha}\right)^{\tau} \\
& =\frac{g}{h} \frac{\omega(1-\delta)(1-g-h)}{(\alpha-\delta g)}
\end{aligned}
$$

Line (58) follows from the definition of $\varphi_{q, t+1}$ and the state equation for $s_{t+1}$ together with the following expressions for the compound returns:

$$
\begin{aligned}
J_{t+1, \tau} & =\delta^{\tau-t-1} \frac{s_{\tau}}{s_{t+1}} \\
R_{t, \tau} & =g^{t-\tau} \alpha^{\tau-t} \frac{k_{\tau+1}}{k_{t+1}} .
\end{aligned}
$$

Line (59) uses $g c_{\tau}=(1-g-h) k_{\tau+1}$. Line (60) follows by simplification. Line (61) uses $k_{t+1} / s_{t+1}=g / h$. Line (63) uses the boundedness of the policy assumption.

\section{Lemma 4}

Allocation $(\mathbf{c}, \mathbf{q}, \mathbf{k})=\left\{c_{t}, q_{t}, k_{t}\right\}_{t=1}^{\infty}$ is Planner-equivalent if it maximizes the utility aggregator

$$
U(\mathbf{c}, \mathbf{q}, \mathbf{k})=\sum_{t=1}^{\infty} \alpha_{t}^{\prime}\left[u\left(c_{t}\right)+v\left(s_{t}\right)\right]
$$

for some utility weights $\alpha_{t}^{\prime} \geq 0$, satisfying $\sum_{t=1}^{\infty} \alpha_{t}^{\prime}<\infty$. In the text, we constructed $\alpha_{t}^{\prime}=\gamma^{t-1}$ as the equilibrium utility weights from the Euler equation for consumption. We prove the necessecity and sufficiency of the cost-benefit rule by proving the following:

Lemma 7 The strictly positive allocation $(\mathbf{c}, \mathbf{q}, \mathbf{k})$ with $\lim _{t \rightarrow \infty} \frac{c_{t+1}}{c_{t} R_{t, t+1}}<1$ is Plannerequivalent if and only if the cost-benefit ratio (54) holds in all periods.

Proof. The if-part of the lemma is straightforward. Once the utility weights are constructed through $\alpha_{t+1}^{\prime}=\frac{u_{t}^{\prime}}{u_{t+1}^{\prime} R_{t, t+1}} \alpha_{t}^{\prime}=\frac{c_{t+1}}{c_{t} R_{t, t+1}} \alpha_{t}^{\prime}$, the condition $\lim _{t \rightarrow \infty} \frac{c_{t+1}}{c_{t} R_{t, t+1}}<1$ ensures 
the bounded mass condition $\sum_{t=1}^{\infty} \alpha_{t}^{\prime}<\infty$, and if first-order conditions are satisfied, the allocation must be Planner equivalent. Consider then the only-if -part, and optimal utility sequence $\left\{u_{t}^{*}+v_{t}^{*}\right\}_{t \geq 1}$ that maximizes $U(\mathbf{c}, \mathbf{q}, \mathbf{k})$. Strict concavity of utility and production functions means that for any non-zero $\left\{\Delta_{t}\right\}_{t=1}^{\infty}$ with $\sum_{t=1}^{\infty} \alpha_{t}^{\prime} \Delta_{t} \geq 0$, $\left\{u_{t}^{*}+v_{t}^{*}+\Delta_{t}\right\}_{t \geq 1}$ is infeasible as utility sequence. For $\sum_{t=1}^{\infty} \alpha_{t}^{\prime} \Delta_{t}<0$, there is a $\varepsilon>0$ such that $u^{*}+v^{*}+\varepsilon \Delta$ is feasible as utility vector. We notice that the first order condition for $k_{t+1}$ defines the (direction of) perturbations $d c_{t}, d c_{t+1}, d k_{t+1}$ that are consistent with perturbations in utility pairs $\left(d u_{t}, d u_{t+1}\right)$ such that $\alpha_{t}^{\prime} d u_{t}+\alpha_{t+1}^{\prime} d u_{t+1}=0$. That is, if we have a $\Delta_{t}$ with $\sum_{t=1}^{\infty} \alpha_{t}^{\prime} \Delta_{t}<0$, then we can construct a sequence of perturbations $d c_{t}, d k_{t+1}$ such that the associated change in utility satisfies $d u_{t} \geq \varepsilon \Delta_{t}$.

If the first-order condition for $q_{t}$ is not met, then there is a feasible perturbation $d q_{t},\left(d s_{\tau}\right)_{\tau=t+1}^{\infty}$ such that the resulting $d u_{t},\left(d v_{\tau}\right)_{\tau=t+1}^{\infty}$ satisfies $\alpha_{t}^{\prime} d u_{t}+\sum_{\tau=t+1}^{\infty} \alpha_{\tau}^{\prime} d v_{\tau}>0$. Now take $\Delta_{t}=-d u_{t}$, and $\Delta_{\tau}=-d v_{\tau}$, and we thus construct a perturbation $d c_{t}, d k_{t+1}$ such that the associated change in utility satisfies $d u_{\tau} \geq \varepsilon \Delta_{\tau}$ for $\tau=t, \ldots, \infty$. If we now add $\varepsilon$ times the perturbation in $q_{t},\left(s_{\tau}\right)_{\tau=t+1}^{\infty}$, we have a feasible perturbation that substitutes capital for public investment, or other way around, and that strictly increases the utility path.

As our $(g, h)$-policy satisfies the condition in the Lemma, the result is proved.

\section{Lemma 5}

When the allocation maximizes the objective

$$
W(\mathbf{c}, \mathbf{q}, \mathbf{k})=\sum_{t=1}^{\infty} \alpha_{t} w_{t}
$$

with non-negative welfare weights $\alpha_{t}$ having a bounded mass $\sum_{t=1}^{\infty} \alpha_{t}<\infty$, then the allocation is Pareto efficient and welfare aggregator $W(\mathbf{c}, \mathbf{q}, \mathbf{k})$ is the objective of a representative Planner.

The equilibrium implies geometric utility weights $\alpha_{t}^{\prime}=\gamma^{t-1}$. If $\gamma<\rho$ or $\gamma<\theta$ one cannot construct a sequence of non-negative welfare weights $\alpha_{t}$ consistent with the sequence of utility weights $\alpha_{t}^{\prime}$. Suppose the contrary, that welfare weights $\alpha_{t} \geq 0$ consistent with $\alpha_{t}^{\prime}$ exist. Then, using the definition of welfare, we see that for some $\tau>t$, the relationship between the two is $\alpha_{1}^{\prime}=\alpha_{1}$ and $\alpha_{\tau}^{\prime}=\sum_{t=1}^{\tau} \alpha_{t} \rho \theta^{\tau-t-1}$ for $\tau>1$. Expanding the latter gives

$$
\alpha_{\tau}^{\prime}=\alpha_{1} \rho \theta^{\tau-2}+\alpha_{2} \rho \theta^{\tau-3}+\ldots+\alpha_{\tau-1} \rho+\alpha_{\tau}
$$


If $\gamma<\theta$ and $\alpha_{1}>0$, we see that the equation cannot hold with $\alpha_{\tau} \geq 0$ for sufficiently large $\tau$. If $\gamma<\rho$, we can write from (64)

$$
\alpha_{\tau+1}^{\prime} \geq \rho \alpha_{\tau}+\alpha_{\tau+1},
$$

or

$$
\gamma \alpha_{\tau}^{\prime}-\rho \alpha_{\tau} \geq \alpha_{\tau+1}
$$

Again, since $\gamma<\rho$, this cannot hold with $\alpha_{\tau+1} \geq 0$ for sufficiently large $\tau$.

Consider now $\gamma \geq \max \{\rho, \theta\}$. We show that now one can construct the non-negative welfare weights. We construct an algorithm for finding the weights. Let $\widetilde{\alpha}_{1}=\left\{\alpha_{\tau}^{1}\right\}_{\tau \geq 1}$, $\widetilde{\alpha}_{2}=\left\{\alpha_{\tau}^{2}\right\}_{\tau \geq 2}$, and so on. Define

$$
\begin{aligned}
\alpha_{\tau}^{1}= & \gamma^{\tau-1}, \tau \geq 1 \\
\alpha_{\tau}^{2}= & \alpha_{\tau}^{1}-\alpha_{1}^{1} \theta^{\tau-t-1}, \tau \geq 2 \\
& \cdots \\
\alpha_{\tau}^{t+1}= & \alpha_{\tau}^{t}-\alpha_{t}^{t} \theta^{\tau-t-1}, \tau \geq t .
\end{aligned}
$$

The value of $\alpha_{\tau}^{t}$ measures the weight remaining for generation $\tau$ after all altruistic weights of generations 1 to $t-1$ have been subtracted. Note that the equilibrium implies utility weights $\widetilde{\alpha}_{1}$, and $\left\{\alpha_{t}^{t}\right\}_{t \geq 1}$ is the sequence of welfare weights consistent with $\widetilde{\alpha}_{1}$. The main intermediate result that we need, in order to prove that the sequence of welfare weights $\left\{\alpha_{t}^{t}\right\}_{t \geq 1}$ is non-negative, is that for all $\tau \geq t$ :

$$
\frac{\alpha_{\tau+1}^{t}}{\alpha_{\tau}^{t}}>\max \{\rho, \theta\}
$$

By construction, this condition is satisfied for $t=1$. It implies that next sequence $\widetilde{\alpha}_{2}$, induced by the algorithm, is non-negative, as

$$
\alpha_{\tau}^{2}=\gamma^{\tau-1}-\theta^{\tau-t-1}>\alpha_{\tau}^{1}\left\{(\max \{\rho, \theta\})^{\tau-1}-\rho \theta^{\tau-t-1}\right\}>0, \tau \geq 2 .
$$

By induction, if the condition holds for $\widetilde{\alpha}_{t}$, the sequence $\widetilde{\alpha}_{t+1}$ is non-negative:

$$
\alpha_{\tau}^{t+1}>\alpha_{\tau}^{t}\left\{(\max \{\rho, \theta\})^{\tau-t}-\rho \theta^{\tau-t-1}\right\}>0, \tau \geq t .
$$

Thus, we are done if we can show that condition (65) holds. Notice that

$$
\alpha_{\tau+1}^{t+1}=\alpha_{\tau+1}^{t}-\alpha_{t}^{t} \rho \theta^{\tau-t}>\max \{\rho, \theta\} \alpha_{\tau}^{t}-\alpha_{t}^{t} \rho \theta^{\tau-t} \geq \theta\left\{\alpha_{\tau}^{t}-\alpha_{t}^{t} \rho \theta^{\tau-t-1}\right\}=\theta\left\{\alpha_{\tau}^{t+1}\right\} .
$$

If $\theta>\rho$, this proves that $\alpha_{\tau+1}^{t+1}>\theta\left\{\alpha_{\tau}^{t+1}\right\}>\rho\left\{\alpha_{\tau}^{t+1}\right\}$. On the other hand, if $\theta<\rho$, we have

$$
\alpha_{\tau+1}^{t+1}=\alpha_{\tau+1}^{t}-\alpha_{t}^{t} \rho \theta^{\tau-t}>\max \{\rho, \theta\} \alpha_{\tau}^{t}-\alpha_{t}^{t} \rho \theta^{\tau-t} \geq \rho\left\{\alpha_{\tau}^{t}-\alpha_{t}^{t} \rho \theta^{\tau-t-1}\right\}=\rho\left\{\alpha_{\tau}^{t+1}\right\},
$$

which completes the proof. 


\section{Lemma 6}

We will show that $\rho<\theta$ gives $\rho<g / \alpha<\theta$, while $\theta<\rho$ results in $\theta<g / \alpha<\rho$. First compare $g / \alpha$ in (45) with $\rho$ :

$$
\begin{aligned}
g / \alpha & <\rho \Leftrightarrow \\
0 & <[\alpha(1-\delta \theta)+\omega(1-\delta)](\rho-\theta) \Leftrightarrow \\
\theta & <\rho
\end{aligned}
$$

The second equivalence follows because all terms between the square brackets are positive. It follows that $\rho<\theta$ gives $\rho<g / \alpha$, while $\theta<\rho$ results in $g / \alpha<\rho$. Now compare $g / \alpha$ and $\theta$ :

$$
\begin{aligned}
g / \alpha & <\theta \Leftrightarrow \\
\rho[1-\delta \theta+\theta \omega(1-\delta)] & <\theta[1-\delta \theta+\rho \omega(1-\delta)+\alpha(1-\delta \theta)(\rho-\theta)] \Leftrightarrow \\
0 & <(\alpha \theta-1)(1-\delta \theta)(\rho-\theta) \Leftrightarrow \\
\rho & <\theta
\end{aligned}
$$

The last equivalence follows because the first term between brackets is negative while the second is positive. This shows the second half of the lemma: $\rho<\theta$ gives $v<\theta$, while $\theta<\rho$ results in $\theta<v$.

\section{Proposition 6}

For the proof of the result, recall that the cost-benefit law does not change the total savings, but only the composition. We will first establish that for given total savings in the benchmark SPE without the cost-benefit requirement the composition of savings maximizes the continuation welfare given by value function $V_{t}$, so that the cost-benefit law must strictly decrease the value of future welfare to the current generation. From Lemma 3 and (36) and (37), one easily derives that the pair $(g, h)$ maximizing $V$ given $g+h=\frac{I}{f(k)}$ for some exogenous $I$ must satisfy $g / h=\xi / \zeta$. As this ratio is preserved in the benchmark SPE, (43) and (44), labeled with BAU, we thus have

$$
V\left(k_{t+1}, s_{t+1} ; g^{C B R}, h^{C B R}\right)<V\left(k_{t+1}, s_{t+1} ; g^{B A U}, h^{B A U}\right)
$$


if $g^{C B R} \neq g^{B A U}$ (Remark 3), where $C B R$ and $B A U$ refer to policies in the two cases. We can then conclude that

$$
\begin{aligned}
w_{t}^{C B R} & =u_{t}^{C B R}+v_{t}^{C B R}+\rho V\left(k_{t+1}^{C B R}, s_{t+1}^{C B R} ; g^{C B R}, h^{C B R}\right) \\
& <u_{t}^{C B R}+v_{t}^{C B R}+\rho V\left(k_{t+1}^{C B R}, s_{t+1}^{C B R} ; g^{B A U}, h^{B A U}\right) \\
& <u_{t}^{B A U}+v_{t}^{B A U}+\rho V\left(k_{t+1}^{B A U}, s_{t+1}^{B A U} ; g^{B A U}, h^{B A U}\right) \\
& =w_{t}^{B A U}
\end{aligned}
$$

The second inequality follows from the fact that welfare without constraints on investments, as in the benchmark SPE, must exceed welfare with additional constraints.

\section{Proposition 7}

Let us denote log-variables by tildes and write the steady state stocks as

$$
\begin{aligned}
\widetilde{k}^{*} & =\frac{\widetilde{g}}{1-\alpha} \\
\widetilde{s}^{*} & =\widetilde{h}+\frac{\alpha}{1-\alpha} \widetilde{g} .
\end{aligned}
$$

Substituting, we can write the steady-state utility level as

$$
u^{*}+v^{*}=\frac{\alpha(1+\omega)}{1-\alpha} \widetilde{g}+\omega \widetilde{h}+\ln (1-g-h),
$$

and consider the investment shares that maximize steady state utility and welfare:

$$
\frac{g^{*}}{h^{*}}=\frac{\alpha}{1-\alpha} \frac{1+\omega}{\omega} .
$$

Let us use $\delta^{*}=\frac{\alpha+\omega}{1+\omega}$ for the critical persistence of the public asset. Comparing the ratio in investments between the benchmark SPE without the cost-benefit rule, denoted by $B A U$, and the steady state optimum, we get

$$
\begin{aligned}
\frac{g^{B A U}}{h^{B A U}} \frac{h^{*}}{g^{*}} & =\frac{\xi}{\zeta} \frac{1-\alpha}{\alpha} \frac{\omega}{1+\omega} \\
& =\frac{1-\alpha \theta-\left(\delta-\delta^{*}\right) \theta(1+\omega)}{1-\alpha \theta} \frac{1-\alpha}{1-\alpha-\left(\delta-\delta^{*}\right)(1+\omega)}
\end{aligned}
$$

where the ratio $\xi / \zeta$ is obtained from the Appendix for the value function. When $\delta=\delta^{*}$, the ratio equals one. This is the case where imposing the cost-benefit rule has no bite since the rule is satisfied anyways; the equilibrium is Planner-equivalent as the persistence of the public asset happens to match the persistence of the other asset. When $\delta>\delta^{*}$, the 
ratio exceeds one and the Planner-equivalence is lost by having too much investment in the neoclassical capital. Imposing the cost-benefit law in this case will further increase investments in the capital stock, at the cost of the public good, and the deviation from the first-best steady-state utility must increase. If the public good is fluid $\left(\delta<\delta^{*}\right)$, the equilibrium investments in capital are too low, and imposing cost-benefit law decreases them further, thereby again increasing the deviation from the first-best steady-state outcome.

Comparing two steady states with utility $u_{A}^{*}+v_{A}^{*}$, and $u_{B}^{*}+v_{B}^{*}$, respectively, the equivalent compensating variation of $B$ relative to $A$, in terms of the consumer good, is directly calculated as $\exp \left(u_{B}^{*}+v_{B}^{*}-u_{A}^{*}-v_{A}^{*}\right)-1$.

\section{References}

[1] Asheim, G. (1988), Rawlsian Intergenerational Justice as a Markov-Perfect Equilibrium in a Resource Technology, Review of Economic Studies LV, 469-484

[2] Barro R.J. (1999), Ramsey meets Laibson in the neoclassical growth model, Quarterly Journal of Economics 114: 1125-1152.

[3] Bassetto, M., and T.J. Sargent (2006), Politics and efficiency of separating capital and ordinary government budgets, Quarterly Journal of Economics, vol.121 n.4, pp.1167-1210.

[4] Bernheim, D.B., and A. Rangel (2009), Beyond Revealed Preference: ChoiceTheoretic Foundations for Behavioral Welfare Economics, The Quarterly Journal of Economics, MIT Press, vol. 124(1), pages 51-104.

[5] Caplin A, and Leahy J (2004), The social discount rate, Journal of Political Economy 112: $1257-1268$.

[6] Dasgupta, P., Discounting Climate Change, Journal of Risk and Uncertainty, 2008, 37(2-3), 141-169.

[7] Glazer, A. (1989), Politics and the Choice of Durability, American Economic Review, $79(5), 1207-1213$.

[8] Gollier, C., Discounting an uncertain future (2002), Journal of Public Economics, 85 (2), pp. 149-166 
[9] Gollier, C., and R. Zeckhauser (2005), Aggregation of Heterogeneous Time Preferences, Journal of Political Economy, 2005, vol. 113, issue 4, ppp. 878-896.

[10] Hanson, S.G., A.K. Kashyap, and J.C. Stein, A Macroprudential Approach to Financial Regulation, Journal of Economic Perspectives, Volume 25, Number 1 (Winter 2011), pp. 3-28.

[11] Karp L. (2005), Global warming and hyperbolic discounting, Journal of Public Economics 89: 261-282.

[12] Karp L. (2007), Non-constant discounting in continuous time, Journal of Economic Theory 132: 557-568.

[13] Krusell P., Kuruscu B, Smith A.A. (2002), Equilibrium welfare and government policy with quasi-geometric discounting, Journal of Economic Theory 105: 42-72.

[14] Laibson D. (1997), Golden eggs and hyperbolic discounting, Quarterly Journal of Economics 112: 443-477.

[15] Lengwiler, Y. (2005), Heterogeneous Patience and the Term Structure of Real Interest Rates, The American Economic Review, 95(3), pp. 890-896.

[16] Lind R.C. (1995), Intergenerational equity, discounting, and the role of cost-benefit analysis in evaluating global climate policy, Energy Policy 23(4-5): 379-389.

[17] Mankiw N.G., D. Romer, and D.N Weil (1992), A contribution to the empirics of economic growth, Quarterly Journal of Economics 107: 407-437.

[18] Nordhaus, W. D.(1993), Optimal greenhouse-gas reductions and tax policy in the "DICE" model, The American Economic Review, 83 (2), pp. 313-317.

[19] Nordhaus, W. D.(2007), A Review of The Stern Review on the Economics of Climate Change, Journal of Economic Literature, 45 (3), 686-702.

[20] O'Donoghue, T., and M. Rabin (1999), Doing It Now or Later, The American Economic Review, Vol. 89, No. 1, pp. 103-124

[21] Phelps E.S. and R.A. Pollak (1968), On second-best national saving and gameequilibrium growth, Review of Economic Studies 35(2): 185-199. 
[22] Persson, T., and L. E. O. Svensson (1989), Why a Stubborn Conservative would Run a Deficit: Policy with Time- Inconsistent Preferences, The Quarterly Journal of Economics, Vol. 104, No. 2 (May, 1989), pp. 325-345.

[23] Rogoff, K. (2010) Three Challenges Facing Modern Macroeconomics, American Economic Association Grand Challenge, White Papers for Future Research, September 21, 2010

[24] Saez-Marti M. and J.W. Weibull (2005), Discounting and altruism to future decision makers, Journal of Economic Theory 122: 254-266

[25] Stern, N. (2006), "The economics of climate change: the Stern review", Cambridge, UK: Cambride University Press.

[26] Strotz, R. H. (1956), Myopia and Inconsistency in Dynamic Utility Maximization, The Review of Economic Studies, 23(3), pp. 165-180.

[27] Tabellini, G, and A. Alesina (1990), Voting on the Budget Deficit, The American Economic Review, Vol. 80, No. 1 (Mar., 1990), pp. 37-49.

[28] Weitzman, M. (2000), Gamma Discounting, The American Economic Review, 91(1), $260-271$.

[29] Weitzman, M. (2007). A Review of The Stern Review on the Economics of Climate Change, Journal of Economic Literature, 45 (3), 703-724. 